\title{
High precision methods for locating the celestial intermediate pole and origin ${ }^{\star}$
}

\author{
N. Capitaine ${ }^{1}$ and P. T. Wallace ${ }^{2}$ \\ 1 Observatoire de Paris, SYRTE/UMR 8630-CNRS, 61 avenue de l'Observatoire, 75014 Paris, France \\ e-mail: n. capitaine@obspm. fr \\ 2 H.M. Nautical Almanac Office, Space Science and Technology Department, CLRC/Rutherford Appleton Laboratory, UK \\ e-mail: P.T.Wallace@rl.ac.uk
}

Received 18 November 2005 / Accepted 11 January 2006

\begin{abstract}
Context. The precession-nutation transformation describes the changing directions on the celestial sphere of the Earth's pole and an adopted origin of right ascension. The coordinate system for the celestial sphere is the geocentric celestial reference system, and the two directions are the celestial intermediate pole (CIP) and the celestial intermediate origin (CIO), the latter having supplanted the equinox for this purpose following IAU resolutions in 2000. The celestial coordinate triad based on the CIP and CIO is called the celestial intermediate reference system; the prediction of topocentric directions additionally requires the Earth rotation angle (ERA), the counterpart of Greenwich sidereal time (GST) in the former equinox based system.

Aims. The purpose of this paper is to review the different ways of calculating the CIP and CIO directions to precisions of a few microarcseconds over a time span of several centuries, meeting the requirements of high-accuracy applications.

Methods. Various implementations are described, their theoretical bases compared and the relationships between the expressions for the relevant parameters are provided. Semi-analytical and numerical comparisons have been made, based on the P03 precession and the IAU 2000A nutation, with slight modifications to the latter to make it consistent with P03.

Results. We have identified which transformations between celestial and terrestrial coordinates involve a minimum number of variables and coefficients for given accuracy objectives. The various methods are consistent at the level of a few microarcseconds over several centuries, and equal accuracy is achievable using both the equinox/GST paradigm and the CIO/ERA paradigm. Given existing nutation models, the most concise expressions for locating the CIP are based on the Fukushima-Williams bias-precession-nutation angles. The CIO can be located to a few microarcseconds using the CIO locator $s$. The equation of the origins (EO) is sensitive to the precession-nutation, but can locate the CIO to a few microarcseconds as long as consistent models are used for EO and precession-nutation.
\end{abstract}

Key words. astrometry - reference systems - ephemerides - celestial mechanics - time

\section{Introduction}

The precession-nutation transformation is a key part of predicting the apparent directions of celestial bodies on a given date, accounting for the changing orientation of the Earth's axis and also defining the zero point for right ascensions ${ }^{1}$ (RA). Since 2003, when IAU Resolutions B1.1-9 came into force, the

$\star$ Tables 5-11 for the series for $s, s+X Y / 2, s+X Y / 2+D, \mathrm{EO}+$ $\Delta \psi \cos \epsilon_{A}, x_{\mathrm{CIO}}, y_{\mathrm{CIO}}$ and $z_{\mathrm{CIO}}$ are only available in electronic form at the CDS, via anonymous ftp to

cdsarc.u-strasbg.fr (130.79.125.5) or via

http://cdsweb.u-strasbg.fr/cgi-bin/qcat?]/A+A/450/855

1 According to the recommendation of the IAU Working Group on nomenclature for fundamental astronomy (NFA WG), the term "right ascension" is used here generically, referring either to an equinox or to the celestial ephemeris origin as required. celestial intermediate pole (CIP) and celestial intermediate origin $(\mathrm{CIO})^{2}$ have been the defining elements in this transformation. The transformation takes directions in the geocentric celestial reference system (GCRS) and expresses them either in the celestial intermediate reference system (CIRS), when using the $\mathrm{CIO}$, or with respect to the true equator and equinox of date, when using the equinox. The position of the CIP at any given date includes the motion due to precession-nutation together with a fixed offset in orientation (of about 23 mas) between the GCRS and J2000 dynamical frame, called the frame bias.

2 The CIO was originally called celestial ephemeris origin (CEO) in the IAU 2000 Resolution B1.8, but is now renamed celestial intermediate origin (CIO) according to the recommendations of the NFA WG for harmonization with the celestial intermediate pole (CIP). 
When directions in the terrestrial intermediate reference frame (TIRS), or equivalently topocentric directions, are to be computed, a measure of Earth rotation is also required, as well as polar motion, which will not be discussed here. When referring right ascensions to the $\mathrm{CIO}$, the appropriate angle is the Earth rotation angle (ERA), a linear function of UT1. In the equinox based case the corresponding angle is Greenwich sidereal time (GST), which is a more complicated function of both UT1 and terrestrial time TT, the latter to deal with part of the precession-nutation effect. The difference between the two angles is called the "equation of the origins" (EO) and is defined as ERA-GST.

Predicting the CIP and CIO directions to precisions of a few microarcseconds over several centuries can be done in a number of ways. Methods based on the IAU 2000A precession-nutation can be found in Capitaine et al. (2003a,b), denoted $\mathrm{C} 03 \mathrm{a}$ and $\mathrm{C} 03 \mathrm{~b}$, respectively, in the following, in the IERS Conventions (2003) and in the IAU SOFA software (Wallace 2002). Methods based on the recent P03 precession model can be found in Capitaine et al. (2003c, 2005), denoted $\mathrm{C} 03 \mathrm{c}$ and $\mathrm{C} 05$. Similarly, various methods exist for computing the bias-precession-nutation transformation (i.e. the product, denoted NPB, of the bias, then precession, then nutation transformations) that either start from or contain implicitly the CIP and CIO. One method is to compute the classical NPB rotation matrix (see Eq. (11)), the bottom row of which is the unit vector of the CIP in the GCRS. Alternatively, direct series for the GCRS $X, Y$ coordinates of the CIP can be derived from the theoretical expressions as functions of the classical precession-nutation angles, and from these coordinates and a small angle $s$ (see below) the rotation matrix can be constructed. Another method is based on the "rotation vector" concept, which was described in $\mathrm{C} 03 \mathrm{c}$ for the bias-precession components and is extended here also to include the nutation.

The classical NPB rotation matrix (see Eq. (11)) can be generated directly from the precession angles and immediately delivers not only the CIP (the bottom row of the matrix) but also the equinox (the top row), which is the RA origin in this case. However, this geometrical simplicity comes at the cost of requiring an Earth rotation measure, namely GST, that itself contains terms due to precession-nutation. Working via the kinematically-defined CIO rather than the equinox means that ERA can be used directly but makes constructing the rotation matrix slightly more complicated given the current conventional form of the nutation. The CIO position can be obtained from the quantity $s(t)$, called the "CIO locator", which is the difference (of less than 0.1 arcsec throughout the period 1900-2100) between the two $R_{3}$ Euler angles in the 3-1-3 sequence of rotations forming the rotation from the GCRS to the CIRS. The computation of $s$ implements the basic kinematical property of the non-rotating origin (NRO, Guinot 1979). Given the initial placement of the $\mathrm{CIO}^{3}$ and the locus of the CIP pole as it moves in the GCRS, the value of $s$ can be expressed as an integral and the results developed in

\footnotetext{
${ }^{3}$ The position of the CIO at epoch, about 0.1 mas from the GCRS prime meridian, was a consequence of the canonical expressions for ERA and GST (C03b).
}

series form for computational convenience. Other implementations of the NRO property are possible either analytically or numerically and expressing positions with respect to different references (Fukushima 2001), such as the GCRS origin axes (Kaplan 2003) or the true equinox (C03c; and Fukushima 2004).

The main purpose of this paper is to compare these various ways of computing the positions of the CIP and CIO and using them to form the transformation between celestial and terrestrial coordinates. The precision goals are a few microarcseconds over a time span of a few hundred years (i.e. about three orders of magnitude better than the expected accuracy of the prediction), meeting the requirements of high-accuracy applications.

All the numerical implementations in the paper will be based upon the $\mathrm{P} 03$ precession model $(\mathrm{C} 03 \mathrm{c})$, which has recently been recommended for adoption as the next IAU standard (Hilton et al. 2006), and the IAU 2000A nutation model, with certain small modifications to the latter to make it conform to the $\mathrm{P} 03$ precession.

We first, in Sect. 2, describe how to construct the GCRS to CIRS transformation starting from the CIP direction and the quantity $s$, or starting from the classical NPB matrix and the EO. Then, in Sect. 3, we describe the methods for highprecision positioning of the CIP, followed by accuracy considerations. In Sect. 4 we go on to describe methods for high precision positioning of the CIO, comparing their theoretical bases, noting the relationship to sidereal time and discussing the accuracy that can be achieved in practice. Finally, in Sect. 5, we report numerical tests of various methods of calculating the CIP and CIO directions and implementing the GCRS to TIRS transformation, and we offer an assessment of which precession-nutation expressions and matrix transformations achieve the required accuracy in the simplest and most efficient way.

\section{The GCRS-to-CIRS matrix}

In this section we review several different ways of constructing the rotation matrix that transforms GCRS vectors into the CIRS. The two conventional methods start from (i) the CIP coordinates $X, Y$ and the CIO locator $s$ and (ii) the classical equinox based NPB matrix and the equation of the origins, respectively. A third option is to use the "rotation-vector" concept.

\subsection{Method based on expressions for $X, Y$ and $s$}

The rotation matrix from the GCRS to the CIRS, defined by the CIP and the CIO, can be written as:

$\mathbf{M}_{\mathrm{CIO}}=R_{3}(-E-s) \cdot R_{2}(d) \cdot R_{3}(E)$,

where $s$ is the quantity locating the CIO in the GCRS and $d, E$ are the GCRS polar coordinates of the CIP defined by:

$$
\begin{aligned}
E & =\arctan (Y / X), \\
d & =\arcsin \left[\left(X^{2}+Y^{2}\right)^{1 / 2}\right] .
\end{aligned}
$$


This can be expressed, equivalently, as:

$\mathbf{M}_{\mathrm{CIO}}=R_{3}(-s) \cdot \mathbf{M}_{\Sigma}$,

$\Sigma$ being the point on the GCRS equator such that $\Sigma_{0} N=\Sigma N$, where $\Sigma_{0}$ is the GCRS $x$-origin and $N$ is the node of the CIP equator on the GCRS equator, and:

$$
\begin{aligned}
& \mathbf{M}_{\Sigma}=\quad R_{3}(-E) \cdot R_{2}(d) \cdot R_{3}(E) \\
& =\left(\begin{array}{ccc}
1-a X^{2} & -a X Y & -X \\
-a X Y & 1-a Y^{2} & -Y \\
X & Y & 1-a\left(X^{2}+Y^{2}\right)
\end{array}\right),
\end{aligned}
$$

with:

$a=1 /(1+\cos d)=1 /(1+Z)=1 /\left[1+\left(1-X^{2}-Y^{2}\right)^{1 / 2}\right]$,

which to an accuracy of $0.1 \mu$ as can be written as:

$a=\frac{1}{2}+\frac{1}{8}\left(X^{2}+Y^{2}\right)+\frac{1}{16}\left(X^{4}+Y^{4}+2 X^{2} Y^{2}\right)$.

Trigonometrical developments for the CIP $X$ and $Y$ coordinates as functions of time can be obtained in a variety of ways, set out in Sect. 3. These can be used directly, or alternatively series for $X$ and $Y$ can be produced, which are of the form:

$$
\begin{aligned}
X= & X_{0}+X_{1} t+X_{2} t^{2}+X_{3} t^{3}+X_{4} t^{4}+X_{5} t^{5} \\
& +\sum_{i} \sum_{j=0}^{3}\left[\left(a_{\mathrm{s}, j}\right)_{i} t^{j} \sin (\mathrm{ARG})+\left(a_{\mathrm{c}, j}\right)_{i} t^{j} \cos (\mathrm{ARG})\right]+\cdots, \\
Y= & Y_{0}+Y_{1} t+Y_{2} t^{2}+Y_{3} t^{3}+Y_{4} t^{4}+Y_{5} t^{5} \\
& +\sum_{i} \sum_{j=0}^{3}\left[\left(b_{\mathrm{c}, j}\right)_{i} t^{j} \cos (\mathrm{ARG})+\left(b_{\mathrm{s}, j}\right)_{i} t^{j} \sin (\mathrm{ARG})\right]+\cdots
\end{aligned}
$$

where ARG stands for various combinations of the fundamental arguments of the nutation theory, including both luni-solar and planetary terms.

The parameter $t$, used in the above expressions as well as in those below, is the elapsed time in Julian centuries since J2000 TT, defined by:

$t=(\mathrm{TT}-2000$ January $1 \mathrm{~d} 12 \mathrm{~h} \mathrm{TT}) / 36525$,

with TT in days.

Note that, for practical reasons, the numerical expressions for $X$ and $Y$ are usually multiplied by the factor $1296000^{\prime \prime} / 2 \pi$ in order to represent the approximate values in arcseconds of the corresponding "angles" (strictly their sines) with respect to the $z$-axis of the GCRS. Note also that the polynomial part of the $X$ and $Y$ CIP coordinates originate from precession, except for the contribution from the frame bias and from cross nutation terms.

\subsection{Method based on the classical equinox based matrix}

Although the ecliptic plays no part in defining either the GCRS or the CIRS, it is nonetheless a basic component of current precession theories. Furthermore, existing nutation theories are based on the ecliptic and equinox of date. A precessionnutation matrix formed by successive rotations using the modeled angles contains not only the CIP but also the equinox. Such a matrix, which we will call $\mathbf{M}_{\text {class, }}$, rotates GCRS vectors into true equator and equinox of date, requiring the use of GST if TIRS coordinates or hour angles are to be computed. Various formulations of $\mathbf{M}_{\text {class }}$ are provided by Eq. (12), Eqs. (14) and (15) in the following subsections.

In order to generate the GCRS to CIRS matrix, a supplementary $R_{3}$ rotation is required, to move the origin from the equinox to the CIO. The angle is called the equation of the origins, and it is equal to ERA-GST:

$\mathbf{M}_{\mathrm{CIO}}=R_{3}(-\mathrm{EO}) \cdot \mathbf{M}_{\text {class }}$.

\subsubsection{Matrix using separate bias, precession and nutation angles}

Using the usual notation, the classical form of the transformation from the GCRS to the CIP and equinox of date system, based on the classical precession variables, can be written as:

$\mathbf{M}_{\text {class }}=\mathbf{N} \cdot \mathbf{P} \cdot \mathbf{B}$,

i.e. as the product of the individual rotation matrices $\mathbf{B}$ (bias) followed by $\mathbf{P}$ (precession) and then $\mathbf{N}$ (nutation):

$$
\begin{aligned}
\mathbf{B} & =R_{1}\left(-\eta_{0}\right) \cdot R_{2}\left(\xi_{0}\right) \cdot R_{3}\left(\mathrm{~d} \alpha_{0}\right), \\
\mathbf{P} & =R_{3}\left(\chi_{A}\right) \cdot R_{1}\left(-\omega_{A}\right) \cdot R_{3}\left(-\psi_{A}\right) \cdot R_{1}\left(\epsilon_{0}\right), \\
\mathbf{N} & =R_{1}\left(-\left[\epsilon_{A}+\Delta \epsilon\right]\right) \cdot R_{3}(-\Delta \psi) \cdot R_{1}\left(\epsilon_{A}\right),
\end{aligned}
$$

where $\xi_{0}$ and $\eta_{0}$ are the celestial pole offsets at $\mathbf{J} 2000$ and $\mathrm{d} \alpha_{0}$ the offset in right ascension of the J2000 mean equatorial frame with respect to the GCRS. The classical precession quantities $\psi_{A}, \omega_{A}, \epsilon_{A}$ and $\chi_{A}$ are those defined by Lieske et al. (1977), the nutation quantities $\Delta \psi$ and $\Delta \epsilon$ are the luni-solar and planetary nutations and $\epsilon_{0}$ is the $\mathbf{J} 2000$ obliquity of the ecliptic.

Note that the precession matrix, $\mathbf{P}$, can be formed in several ways, depending on which of the precession angles are used. The 4-rotation method given above was chosen for the IERS/SOFA implementation of IAU 2000 because it enabled the specified precession-rate adjustments to be applied directly and unambiguously. In the case of the present work that is no longer a consideration and other choices are open.

One particular formulation for $\mathbf{P}$ customarily used in the past but no longer useful is:

$\mathbf{P}=R_{3}\left(-z_{A}\right) \cdot R_{2}\left(\theta_{A}\right) \cdot R_{3}\left(-\zeta_{A}\right)$

The Euler angles it uses, $z_{A}, \theta_{A}$ and $\zeta_{A}$, are further from the basic P03 quantities for the equator $\psi_{A}$ and $\omega_{A}$ used in Eq. (12), and while the method would save one rotation the result would still be much less concise than that described in Sect. 2.2.3 ${ }^{4}$.

\footnotetext{
${ }^{4}$ Although a further three rotations can be saved by absorbing the frame bias into the $z_{A}, \theta_{A}$ and $\zeta_{A}$ angles, two of the resulting angles undergo large changes near epoch that preclude any convenient polynomial representation (see $\mathrm{C} 03 \mathrm{c}$ ).
} 


\subsubsection{Matrix referred to the J2000 ecliptic}

Alternatively, the NPB transformation can be expressed with the nutation angles referred to the J2000 ecliptic:

$$
\begin{aligned}
\mathbf{M}_{\text {class }}^{\prime}=R_{3}\left(\chi_{A}^{\prime}\right) \cdot R_{1}\left(-\omega_{A}^{\prime}\right) & \cdot R_{3}\left(-\psi_{A}^{\prime}\right) \\
& \cdot R_{1}\left(\epsilon_{0}-\eta_{0}\right) \cdot R_{3}\left(\mathrm{~d} \alpha_{0}^{\prime}\right),
\end{aligned}
$$

where $\chi_{A}^{\prime}=\chi_{A}+\Delta \chi_{A}$ is the displacement of the ecliptic along the CIP equator, $\psi_{A}^{\prime}=\psi_{A}+\Delta \psi_{1}+\xi_{0} / \sin \epsilon_{0}$ is the precessionnutation-bias along the $\mathrm{J} 2000$ ecliptic, $\omega_{A}^{\prime}=\omega_{A}+\Delta \epsilon_{1}$ is the inclination of the CIP equator on the J2000 ecliptic and $\mathrm{d} \alpha_{0}^{\prime}=$ $\mathrm{d} \alpha_{0}+\xi_{0} \cot \epsilon_{0}$ is the GCRS right ascension of the intersection of the J2000 ecliptic with the GCRS equator.

This extends the form of the precession-nutation proposed by Aoki \& Kinoshita (1983) by including the bias as well.

Note that the full benefits of this approach (i.e. referring the precession-nutation quantities to a fixed plane) are obtained by omitting the precession of the ecliptic (i.e. $\chi_{A}^{\prime}$ ), which means considering the transformation from GCRS to the system defined by the CIP and the intersection $\gamma_{1}$ of the CIP equator with the J2000 ecliptic instead of the equinox of date.

\subsubsection{Matrix using new precession-nutation angles}

A new formulation has been proposed by Fukushima (2003) to express the precession and nutation as concisely as possible, using four rotations, by extending the notations proposed by Williams (1994) to take account of the bias contribution. This can be written as:

$\mathbf{M}_{\mathrm{FW}}=R_{1}\left(-\epsilon^{\prime}\right) \cdot R_{3}\left(-\psi^{\prime}\right) \cdot R_{1}\left(\phi^{\prime}\right) \cdot R_{3}\left(\gamma^{\prime}\right)$,

where $\epsilon^{\prime}=\left[\epsilon_{A}+\Delta \epsilon\right]$ is the inclination of the ecliptic of date on the CIP equator, $\psi^{\prime}=\left[\psi+\Delta \psi+\xi_{0} / \sin \epsilon_{0}\right]$ the precession plus nutation angle plus bias in longitude along the ecliptic of date, $\phi^{\prime}=\left[\phi+\eta_{0}\right]$ is the obliquity of the ecliptic of date on the GCRS equator and $\gamma^{\prime}=\left[\gamma+\mathrm{d} \gamma_{0}\right]$ is the GCRS right ascension of the intersection of the ecliptic of date with the GCRS equator.

This method is similar to Eq. (14) but with quantities referred to the ecliptic of date instead of the ecliptic at J2000. It obviously results in simpler relationships as the IAU 2000A nutation series provides such quantities directly, but can be regarded as less satisfying from a theoretical point of view.

\subsection{Method based on the "rotation vector" concept}

Any finite rotation of the coordinate frame can be expressed as the "Euler axis and angle", which are, respectively, the unit vector along the axis of rotation and the amount of rotation. These can be combined in various ways (see Goldstein 1980; Wertz 1986) of which a particularly straightforward three-component option is simply to scale the Euler-axis unit vector by the amount of rotation in radians. This "rotation vector" approach was shown in $\mathrm{C} 03 \mathrm{c}$ to be efficient for representing the biasprecession because the precession approximates a constant rotation about a fixed point (namely the ecliptic pole). We have extended it to bias-precession-nutation and applied it both to the equinox based and the CIO based transformation.
To express the vector $\left(x_{r}, y_{r}, z_{r}\right)$ as the rotation matrix $\mathbf{M}_{\mathrm{rot}}$, we first decompose it into the amount of rotation in radians:

$\phi=\left(x_{r}^{2}+y_{r}^{2}+z_{r}^{2}\right)^{1 / 2}$

and the rotation-axis unit vector:

$x=x_{r} / \phi, \quad y=y_{r} / \phi, \quad z=z_{r} / \phi$.

Then, writing $S=\sin \phi, C=\cos \phi$ and $f=1-C$, we form the matrix elements as follows:

$\mathbf{M}_{\mathrm{rot}} \simeq\left(\begin{array}{lll}x x f+C & x y f+S z & x z f-S y \\ y x f-S z & y y f+C & y z f+S x \\ z x f+S y & z y f-S x & z z f+C\end{array}\right)$.

Note that the algorithm is computationally very efficient: only one square root and two trigonometrical functions (of the same angle) are required, in addition to arithmetic operations. Given the date $t$ in Julian centuries after J2000, Eqs. (16) to (18) can be evaluated to generate the matrix transformation for bias, precession and nutation.

The product of this matrix with the GCRS vector gives either the true place of date or the intermediate place, depending on the chosen $x$-origin (i.e. either the equinox or the CIO).

\subsection{Relationship between the methods}

\subsubsection{CIP, $\mathrm{CIO}$ and equinox from matrix elements}

Recalling that GCRS vectors $\boldsymbol{r}_{\mathrm{GCRS}}$ can be transformed into CIRS vectors $\boldsymbol{r}_{\mathrm{CIRS}}$ as follows:

$\boldsymbol{r}_{\mathrm{CIRS}}=\mathbf{M}_{\mathrm{CIO}} \cdot \boldsymbol{r}_{\mathrm{GCRS}}$,

we can write the matrix in terms of unit row vectors $v$ :

$\mathbf{M}_{\mathrm{CIO}} \equiv\left(\begin{array}{c}\boldsymbol{v}_{\mathrm{CIO}} \\ \boldsymbol{v}_{\mathrm{CIP}} \times \boldsymbol{v}_{\mathrm{CIO}} \\ \boldsymbol{v}_{\mathrm{CIP}}\end{array}\right)$

This identifies the top row of the GCRS to CIRS matrix as the CIO vector, the bottom row as the CIP vector and the middle row as the CIRS $y$-axis. The equivalent expressions in the classical case are:

$\boldsymbol{r}_{\text {true }}=\mathbf{M}_{\text {class }} \cdot \boldsymbol{r}_{\mathrm{GCRS}}$,

and:

$\mathbf{M}_{\text {class }} \equiv\left(\begin{array}{c}\boldsymbol{v}_{\text {eqx }} \\ \boldsymbol{v}_{\mathrm{CIP}} \times \boldsymbol{v}_{\mathrm{eqx}} \\ \boldsymbol{v}_{\mathrm{CIP}}\end{array}\right)$,

where the top row $\left(\boldsymbol{v}_{\text {eqx }}\right)$ is the unit vector pointing to the equinox of date.

In particular, note that in both cases the bottom row is the CIP vector, leading to the useful identities:

$X=\mathbf{M}(3,1)$,

$Y=\mathbf{M}(3,2)$. 


\subsubsection{The matrix elements as functions of CIP $X, Y$}

Referring to Eqs. (3), (4) and (10), we recognize that any of the different forms of the matrix transformations, corresponding to various origins on the CIP equator, can be written out in a similar way:

$\mathbf{M}_{\beta}=R_{3}(-\beta) \cdot \mathbf{M}_{\Sigma}$,

where $\mathbf{M}_{\Sigma}$ is given by Eq. (4) and $\beta$ is the rotation angle from $\Sigma$ to the origin of the selected intermediate origin on the CIP equator, so that:

$\beta=0$ for the origin $\Sigma$,

$\beta=s$ for the $\mathrm{CIO}$ based matrix and

$\beta=-\mathrm{EO}+s$ for the equinox based matrix.

The $\mathbf{M}(i, j)$ elements of the matrix transformation $\mathbf{M}_{\beta}$ as functions of the $X, Y$ coordinates and the $\beta$ angle can be derived from Eqs. (4) and (24) as follows:

$$
\begin{aligned}
& \mathbf{M}(1,1)=\left(1-a X^{2}\right) \cos \beta+a X Y \sin \beta, \\
& \mathbf{M}(1,2)=-a X Y \cos \beta-\sin \beta\left(1-a Y^{2}\right), \\
& \mathbf{M}(1,3)=-\cos \beta X+\sin \beta Y, \\
& \mathbf{M}(2,1)=\left(1-a X^{2}\right) \sin \beta-a X Y \cos \beta, \\
& \mathbf{M}(2,2)=-a X Y \sin \beta+\cos \beta\left(1-a Y^{2}\right), \\
& \mathbf{M}(2,3)=-\sin \beta X-\cos \beta Y, \\
& \mathbf{M}(3,1)=X, \\
& \mathbf{M}(3,2)=Y, \\
& \mathbf{M}(3,3)=1-a\left(X^{2}+Y^{2}\right) .
\end{aligned}
$$

These expressions underline the fact that locating the pole and the origin of right ascension are essentially different topics, and we deal with each of them in Sects. 3 and 4 respectively.

\subsubsection{The rotation vector components expressed as functions of the other quantities}

In Eq. (18), we expanded the $r$-vector representation of the GCRS to CIRS transformation into the nine elements of the NPB matrix $\mathbf{M}_{\text {rot }}$. Elements $(3,1)$ and $(3,2)$ provide the following expressions for the GCRS CIP coordinates $X$ and $Y$ as functions of the components of the rotation-axis unit vector, $x$, $y, z$, and the amount of rotation $\phi$ around the Euler axis:

$X=\mathbf{M}(3,1)=z x(1-\cos \phi)+\sin \phi y$,

$Y=\mathbf{M}(3,2)=z y(1-\cos \phi)-\sin \phi x$.

Conversely, the components of the rotation-axis unit vector, $x, y, z$, can be derived from the differences between the elements of the GCRS-to-intermediate-system transformation matrix, which are such that:

$\mathbf{M}(3,1)-\mathbf{M}(1,3)=2 S y$,

$\mathbf{M}(3,2)-\mathbf{M}(2,3)=-2 S x$,

$\mathbf{M}(2,1)-\mathbf{M}(1,2)=-2 S z$.
The expressions for $x, y, z$ as functions of $X, Y$ and the generalized rotation angle $\beta$ are:

$S x=-Y-\frac{1}{2}[(\cos \beta-1) Y+\sin \beta X]$,

$S y=X+\frac{1}{2}[(\cos \beta-1) X-\sin \beta Y]$,

$S z=\left(1-\frac{1}{2} a\left(X^{2}+Y^{2}\right)\right] \sin \beta$.

The rotation vector components $x_{r}, y_{r}, z_{r}$ can then be derived from the previous expressions by using the relations

$x_{r}=(\phi / \sin \phi) S x$,
$y_{r}=(\phi / \sin \phi) S y$,
$z_{r}=(\phi / \sin \phi) S z$,

the expression $\phi / \sin \phi$ being obtained from the matrix elements:

$\phi / \sin \phi=1+\frac{1}{6} S_{2}+\frac{3}{40} S_{2}^{2}+\frac{169}{5040} S_{2}^{3}$

with:

$$
\begin{aligned}
S_{2}= & \frac{1}{4}\left([\mathbf{M}(3,1)-\mathbf{M}(1,3)]^{2}+[\mathbf{M}(3,2)-\mathbf{M}(2,3)]^{2}\right. \\
& \left.+[\mathbf{M}(2,1)-\mathbf{M}(1,2)]^{2}\right) \\
= & S^{2}\left(x^{2}+y^{2}+z^{2}\right)=\sin ^{2} \phi .
\end{aligned}
$$

This applies (i) to the CIO based case with $\beta=s$ and (ii) to the equinox based case with $\beta=-\mathrm{EO}+s$. As $\beta$ is a small angle and $S_{2}$ a quantity of the order of $10^{-4}$, we have:

$$
\begin{aligned}
& x_{r} \simeq-Y-\frac{1}{2} \beta X \\
& y_{r} \simeq X-\frac{1}{2} \beta Y \\
& z_{r} \simeq \beta,
\end{aligned}
$$

which shows that the rotation-vector components $x_{r}, y_{r}, z_{r}$ are close to $-Y, X, \beta$.

\section{The position of the CIP}

Three methods of generating the CIP coordinates will be examined, one as used for the published $X, Y$ series and two based on different choices of precession and nutation angles.

\subsection{Method used for the IERS $X, Y$ series}

IERS Conventions (2003) present direct series for $X$ and $Y$, providing convenient access to accurate predictions while minimizing the number of opportunities for incomplete or incorrect implementation. The starting point for developing these series is to write the position of the CIP directly as the components $X(t)=\sin d \cos E, Y(t)=\sin d \sin E$ of the unit vector in the GCRS, $d$ and $E$ being the GCRS polar coordinates of the CIP and $Z=\cos d$. 
The expressions for the precession-nutation contributions $\bar{X}$ and $\bar{Y}$ to $X$ and $Y$ as functions of the precession and nutation quantities, $\psi=\psi_{A}+\Delta \psi_{1}$ and $\omega=\omega_{A}+\Delta \epsilon_{1}$, referred to the J2000 ecliptic are:

$\bar{X}=\sin \omega \sin \psi$,

$\bar{Y}=-\sin \epsilon_{0} \cos \omega+\cos \epsilon_{0} \sin \omega \cos \psi$,

$X, Y$ being related to $\bar{X}, \bar{Y}$ by:

$X=\bar{X}+\xi_{0}-\mathrm{d} \alpha_{0} \bar{Y}$,

$Y=\bar{Y}+\eta_{0}+\mathrm{d} \alpha_{0} \bar{X}$,

where $\xi_{0}$ and $\eta_{0}$ are the celestial pole offsets at J2000 and d $\alpha_{0}$ the offset in right ascension of the J2000 mean equatorial frame with respect to the GCRS. Semi-analytical expressions for the quantities $X$ and $Y$ have been developed in C03a and C03c, using Eqs. (33) and (34), that are consistent with the IAU 2000A nutation series and the IAU 2000A and P03 precession solutions respectively. These expressions are based on the MHB2000 values (Mathews et al. 2002) for the celestial pole offsets at J2000 (i.e. $\xi_{0}=-16.617$ mas and $\eta_{0}=-6.819$ mas) and on the value of Chapront et al. (2002) for $\mathrm{d} \alpha_{0}$ (i.e. $\left.-14.6 \mathrm{mas}\right)$.

To transform the nutation angles $\Delta \psi$ and $\Delta \epsilon$ referred to the ecliptic of date to angles $\Delta \psi_{1}, \Delta \epsilon_{1}$ referred to the $\mathrm{J} 2000$ ecliptic, the following relations were used:

$$
\begin{aligned}
\Delta \psi_{1} \sin \epsilon_{1} & =\Delta \psi \sin \epsilon \cos \chi_{A}-\Delta \epsilon \sin \chi_{A}, \\
\Delta \epsilon_{1} & =\Delta \epsilon \cos \chi_{A}+\Delta \psi \sin \epsilon \sin \chi_{A} .
\end{aligned}
$$

The classical precession quantities $\psi_{A}, \omega_{A}, \epsilon_{A}$ and $\chi_{A}$ are those defined by Lieske et al. (1977) $\left(\psi_{A}, \omega_{A}\right.$ being the basic P03 quantities for the precession of the equator) and the nutation quantities $\Delta \psi$ and $\Delta \epsilon$ are the luni-solar and planetary nutations.

\subsection{Methods using the equinox based matrix elements}

\subsubsection{Method based on the $M_{\text {class }}$ form of the NPB matrix}

Elements $(3,1)$ and $(3,2)$ of the form $\mathbf{M}_{\text {class }}$ of the NPB matrix (cf. Eq. (11)) provide expressions for the GCRS CIP coordinates $X, Y$ as functions of the classical precession quantities, $\psi_{A}, \omega_{A}$ and $\chi_{A}$, nutation quantities $\Delta \psi$ and $\Delta \epsilon$, the J2000 obliquity of the ecliptic $\epsilon_{0}$ and the frame biases $\xi_{0}$, $\eta_{0}$ and $\mathrm{d} \alpha_{0}$. Omitting the frame biases for simplification and using the intermediary quantities $x_{1}=\sin \left(\epsilon_{A}+\Delta \epsilon\right) \sin \Delta \psi$, $x_{2}=\sin \left(\epsilon_{A}+\Delta \epsilon\right) \cos \Delta \psi$ and $y=\cos \left(\epsilon_{A}+\Delta \epsilon\right)$ leads to the following expressions:

$$
\begin{aligned}
X= & \mathbf{M}(3,1) \\
= & x_{1}\left(\cos \chi_{A} \cos \psi_{A}+\sin \chi_{A} \cos \omega_{A} \sin \psi_{A}\right) \\
& +\left(x_{2} \cos \epsilon_{A}-y \sin \epsilon_{A}\right) \\
& \times\left(-\sin \chi_{A} \cos \psi_{A}+\cos \chi_{A} \cos \omega_{A} \sin \psi_{A}\right) \\
& +\left(x_{2} \sin \epsilon_{A}+y \cos \epsilon_{A}\right) \sin \omega_{A} \sin \psi_{A}
\end{aligned}
$$

$$
\begin{aligned}
Y= & \mathbf{M}(3,2) \\
= & x_{1}\left[\left(-\cos \chi_{A} \sin \psi_{A}+\sin \chi_{A} \cos \omega_{A} \cos \psi_{A}\right) \cos \epsilon_{0}\right. \\
& \left.+\sin \chi_{A} \sin \omega_{A} \sin \epsilon_{0}\right] \\
& +\left(x_{2} \cos \epsilon_{A}-y \sin \epsilon_{A}\right) \\
& \times\left[\left(\sin \chi_{A} \sin \psi_{A}+\cos \chi_{A} \cos \omega_{A} \cos \psi_{A}\right) \cos \epsilon_{0}\right. \\
& \left.+\cos \chi_{A} \sin \omega_{A} \sin \epsilon_{0}\right]+\left(x_{2} \sin \epsilon_{A}+y \cos \epsilon_{A}\right) \\
& \times\left(\sin \omega_{A} \cos \psi_{A} \cos \epsilon_{0}-\cos \omega_{A} \sin \epsilon_{0}\right) .
\end{aligned}
$$

This way of expressing the CIP position is not straightforward, even in the simplified case where the frame biases are omitted, due to the fact that it mixes precession quantities referred to the J2000 ecliptic and nutation quantities referred to the ecliptic of date. The advantage is that the precession quantities are the basic P03 quantities and the nutation quantities are the basic IAU 2000A ones.

A simpler form can be developed using nutation quantities referred to the $\mathrm{J} 2000$ ecliptic. The $X, Y$ expressions derived from the $\mathbf{M}_{\text {class }}^{\prime}$ form of the NPB matrix are:

$$
\begin{aligned}
X= & \mathbf{M}(3,1) \\
= & \sin \omega_{A}^{\prime} \sin \psi_{A}^{\prime} \cos \mathrm{d} \alpha_{0}^{\prime}-\left[\sin \omega_{A}^{\prime} \cos \psi_{A}^{\prime} \cos \left(\epsilon_{0}-\eta_{0}\right)\right. \\
& \left.\quad-\cos \omega_{A}^{\prime} \sin \left(\epsilon_{0}-\eta_{0}\right)\right] \sin \mathrm{d} \alpha_{0}^{\prime}, \\
Y= & \mathbf{M}(3,2) \quad \\
= & \sin \omega_{A}^{\prime} \sin \psi_{A}^{\prime} \sin \mathrm{d} \alpha_{0}^{\prime}+\left[\sin \omega_{A}^{\prime} \cos \psi_{A}^{\prime} \cos \left(\epsilon_{0}-\eta_{0}\right)\right. \\
& \left.-\cos \omega_{A}^{\prime} \sin \left(\epsilon_{0}-\eta_{0}\right)\right] \cos \mathrm{d} \alpha_{0}^{\prime} .
\end{aligned}
$$

with the same notations as in Sect. 2.2.2. Note that even though Eq. (37) is much simpler than Eq. (36), this is not the simplest way of expressing the CIP position given the form of the current IAU nutation series, which are referred to the ecliptic of date. The nutation quantities referred to the J2000 ecliptic should instead be deduced from the IAU nutation (e.g. through Eq. (35)). However, the precession quantities used in this expression are the P03 basic quantities and, from a theoretical standpoint, quantities referred to a fixed ecliptic are more compliant with the use of the GCRS; new solutions for nutation may be provided in this form, making the above relationship interesting for future applications.

\subsubsection{Method based on the $M_{\mathrm{FW}}$ form of the NPB matrix}

As before, elements $(3,1)$ and $(3,2)$ of the form $\mathbf{M}_{\mathrm{FW}}$ (cf. Eq. (15)) of the NPB matrix provide expressions for the GCRS CIP coordinates $X, Y$ as functions of the new precessionnutation-bias quantities referred to the ecliptic of date and of the $\mathrm{J} 2000$ obliquity of the ecliptic $\epsilon_{0}$.

These can be written as (Fukushima 2003):

$$
\begin{aligned}
X= & \mathbf{M}(3,1) \\
= & \sin \epsilon^{\prime} \sin \psi^{\prime} \cos \gamma^{\prime}-\left(\sin \epsilon^{\prime} \cos \psi^{\prime} \cos \phi^{\prime}\right. \\
& \left.\quad-\cos \epsilon^{\prime} \sin \phi^{\prime}\right) \sin \gamma^{\prime}, \\
Y= & \mathbf{M}(3,2) \quad \\
= & \sin \epsilon^{\prime} \sin \psi^{\prime} \sin \gamma^{\prime}+\left(\sin \epsilon^{\prime} \cos \psi^{\prime} \cos \phi^{\prime}\right. \\
& \left.\quad-\cos \epsilon^{\prime} \sin \phi^{\prime}\right) \cos \gamma^{\prime} .
\end{aligned}
$$


These expressions are similar to Eq. (37) but with the precession-nutation angles being referred to the ecliptic of date instead of the J2000 ecliptic. This is an advantage as these angles are the quantities directly provided by the IAU 2000A nutation series. However, this can be regarded as less satisfying from a theoretical point of view and therefore may not suit future applications. Another possible objection is that the precession quantities used are not the basic $\mathrm{P} 03$ precession quantities but are derived quantities.

\subsection{Relationships between the methods}

Any form of the GCRS CIP coordinates $X, Y$ obtained in the previous sections are functions of the precession-nutationbias quantities, which are time polynomials, Poisson series, and constant terms, respectively. Three different expressions, namely Eq. (34) of Sect. 3.1, Eq. (37) of Sect. 3.2.1 and Eq. (38) of Sect. 3.2.2 have been developed into series by using the software package GREGOIRE (Chapront 2003), which carries out Poisson series manipulations (for further details, see C03a). Those expressions differ in the way the bias and precession-nutation contributions are treated, the consequences of which on the CIP location are studied in the following sections.

\subsubsection{Differences in the bias contribution}

The bias contribution is interpreted in different ways in the method used for the $X, Y$ series in Sect. 3.1 and the method using the elements of the NPB matrix in Sect. 2.2.1.

In the Sect. $3.1 X, Y$ series, the celestial pole offsets $\xi_{0}$ and $\eta_{0}$ have been interpreted simply as constant terms added to the precession-nutation expressions, $\bar{X}$ and $\bar{Y}$.

The contributions $\mathrm{d} X_{\text {bias }}$ and $\mathrm{d} Y_{\text {bias }}$ for the IAU 2000 frame biases to the Sect. 3.1 X, $Y$ series are, in $\mu$ as:

$$
\begin{aligned}
& \mathrm{d} X_{\text {bias }}=-16617-1.6 t^{2}+1 \cos \Omega, \\
& \mathrm{d} Y_{\text {bias }}=-6819-142 t+1 \sin \Omega,
\end{aligned}
$$

the first term in each coordinate being the contribution from the celestial pole offset at J2000 and the two following ones from the frame bias in right ascension.

It can be shown that if the celestial pole offsets, $\xi_{0}$ and $\eta_{0}$, are interpreted simply as constant terms added to the precession-nutation expressions, $\bar{X}$ and $\bar{Y}$, this differs from applying them through a frame bias rotation matrix, by $-0.78 \mu$ as $t^{2}$ in $X$ and $-0.33 \mu$ as $t^{2}$ in $Y$.

These differences are seen when comparing the $X, Y$ series Eqs. (48) and (49) with respect to series based on Eqs. (37) or (38) for elements $\mathbf{M}(3,1)$ and $\mathbf{M}(3,2)$ of the NPB matrix.

\subsubsection{Differences in the precession-nutation contribution}

There are differences in the way precession-nutation is considered in the various methods described in Sects. 3.1, 3.2.1 and 3.2.2. For precession, either expressions for the basic P03 quantities $\psi_{A}$ and $\omega_{A}$, plus the quantity $\chi_{A}$ in the case of
Eq. (35), are used (in the methods of Sects. 3.1 and 3.2.1), or the result of applying the $\mathrm{P} 03$ precession to the F-W angles (in the method of Sect. 3.2.2). This result was derived in a semianalytical way, by solving to microarcsecond accuracy the P03 expressions for the F-W angles, using spherical-triangle formulas and the developments for the basic P03 angles.

The nutation quantities are considered either as nutation angles referred to the ecliptic of date (first method of Sect. 3.2.1 and the method of Sect. 3.2.2), or are transformed into nutation angles referred to the J2000 ecliptic (method of Sect. 3.1 and the second method of Sect. 3.2.1).

Transforming the nutation angles from the ecliptic of date to the J2000 ecliptic by using Eq. (35) is not totally rigorous, involving approximations at a submicroarcsecond level. Other approximations at the same level were also used in the semianalytical developments of the precession-nutation quantities performed in Sect. 3.1.

Those approximations can be assessed by comparing the Sect. $3.1 X, Y$ series with respect to series of the $(3,1)$ and $(3,2)$ elements of the NPB matrix based on the nutation angles referred to the ecliptic of date, for example using Eq. (38). The largest differences (in addition to those mentioned in Sect. 3.3.1) that are seen in this comparison are of $0.22 \mu$ as $t \sin 2 \Omega$ in $X$ and $-0.12 \mu$ as $t$ in $Y$, which result from the approximations of Eq. (35).

\subsection{Accuracy considerations}

\subsubsection{Dependence on the parameters of the precession model}

The GCRS $X, Y$ coordinates of the CIP and, similarly, the classical precession-nutation quantities, are functions of the parameters that were used to develop the precession model. Both depend on the integration constants (i.e. the linear terms $X_{1}$ and $Y_{1}$ for the $X, Y$ case, the precession rates in the classical case) and the value for the secular variation of the Earth's dynamical flattening (or equivalently the secular variation of $J_{2}$ ), one of the precession model parameters due to non-rigidity.

In the case of the expressions for the GCRS coordinates of the CIP, irrespective of how they are computed, adjustments to the above parameters of the precession model and additionally to the frame bias components $\xi_{0}, \eta_{0}$ and $\mathrm{d} \alpha_{0}$ cause changes in $X$ and $Y$ as follows:

$$
\begin{aligned}
\mathrm{d} X= & \mathrm{d} \xi_{0}+\mathrm{d} X_{1} t \\
& +0.0001 \mathrm{~d}\left(\mathrm{~d} \alpha_{0}\right) t^{2}+0.0203 \mathrm{~d} Y_{1} t^{2} \\
& +\left(\dot{J}_{2} / J_{2}\right) \times\left(1002^{\prime \prime} 5 t^{2}-0 .{ }^{\prime} 4 t^{3}\right)
\end{aligned}
$$

$$
\begin{aligned}
\mathrm{d} Y= & \mathrm{d} \eta_{0}+X_{1} \mathrm{~d}\left(\mathrm{~d} \alpha_{0}\right) t+\mathrm{d} Y_{1} t \\
& -0.0224 \mathrm{~d} X_{1} t^{2}-\left(\dot{J}_{2} / J_{2}\right) \times\left(22^{\prime \prime} .5 t^{3}\right) .
\end{aligned}
$$

In particular, the change from the IAU 2000 precession solution to the $\mathrm{P} 03$ solution gives rise to changes in the polynomial terms in the $X$ and $Y$ series of, in $\mu$ as:

$$
\begin{aligned}
& \mathrm{d} X=155 t-2564 t^{2}+2 t^{3}+54 t^{4} \\
& \mathrm{~d} Y=-514 t-4 t^{2}+58 t^{3}-1 t^{4}-1 t^{5}
\end{aligned}
$$


the $t^{4}$ and $t^{5}$ terms being absent from the IAU 1976/2000 precession. There are also changes of the order of a few $\mu$ as per century in a few terms of the periodic part of the expressions for the CIP $X, Y$ due to the $J_{2}$ rate effect which is taken into account in the $\mathrm{P} 03$ precession model whereas it was not considered in the IAU 2000 model. The additional Poisson terms to be considered are proportional to $\dot{J}_{2} / J_{2}$. These terms, derived from the corresponding terms in longitude and obliquity provided in $\mathrm{C} 05$, are, as functions of $\dot{J}_{2} / J_{2}$, in arcseconds:

$$
\begin{aligned}
\mathrm{d} X_{\mathrm{J} 2 \mathrm{~d}}= & \left(\dot{J}_{2} / J_{2}\right) t[-6.8 \sin \Omega-5.2 \sin 2(F-D+\Omega) \\
& -0.1 \sin 2(F+\Omega)+0.1 \sin 2 \Omega], \\
\mathrm{d} Y_{\mathrm{J} 2 \mathrm{~d}}= & \left(\dot{J}_{2} / J_{2}\right) t[9.2 \cos \Omega+0.6 \cos 2(F-D+\Omega)] .
\end{aligned}
$$

The $J_{2}$ rate contribution to the linear precession rate in longitude, which in the $\mathrm{P} 03$ precession was considered to be $-14 t$ mas, corresponds to a value for $\dot{J}_{2} / J_{2}$ of $-2.7774 \times$ $10^{-6} / \mathrm{cy}$, or equivalently (with $J_{2}=1.0826358 \times 10^{-3}$ ) to a value for $\dot{J}_{2}$ of $-0.3001 \times 10^{-9} /$ cy.

In the case of the classical precession-nutation quantities, adjustments $\mathrm{d} r_{0}$ and $\mathrm{d} u_{0}$ to the precession rates in longitude and obliquity and the introduction of a $J_{2}$ rate contribution cause changes in the basic precession quantities $\psi_{A}$ and $\omega_{A}$ of:

$$
\begin{aligned}
\mathrm{d} \psi_{A}= & \mathrm{d} r_{0} t-0.0053 \mathrm{~d} u_{0} t^{2} \\
& +\left(\dot{J}_{2} / J_{2}\right) \times\left(2520 .^{\prime} 4 t^{2}-0 .^{\prime} 9 t^{3}\right) \\
\mathrm{d} \omega_{A}= & \mathrm{d} u_{0} t .
\end{aligned}
$$

Two different corrections in nutation have to be considered when changing from the IAU 2000 precession solution to the P03 solution.

It is first necessary to take into account the additional Poisson terms, proportional to $\dot{J}_{2} / J_{2}$, which have already been mentioned in the $X, Y$ case and which affect the nutation amplitudes in both longitude and obliquity. The corrections larger than $1 \mu$ as to be added to the IAU 2000A nutation for this effect are, in $\mu$ as:

$$
\begin{aligned}
\mathrm{d} \psi_{\mathrm{J} 2 \mathrm{~d}}= & +47.8 t \sin \Omega+3.7 t \sin 2(F-D+\Omega) \\
& +0.6 t \sin 2(F+\Omega)-0.6 t \sin 2 \Omega, \\
\mathrm{d} \varepsilon_{\mathrm{J} 2 \mathrm{~d}}= & -25.6 t \cos \Omega-1.6 t \cos 2(F-D+\Omega),
\end{aligned}
$$

which can also be written as functions of $\Delta \psi_{\text {IAU2000 }}$ and $\Delta \varepsilon_{\text {IAU2000, }}$, the IAU 2000A nutation angles in longitude and obliquity respectively:

$$
\begin{aligned}
\mathrm{d} \psi_{\mathrm{J} 2 \mathrm{~d}} & =\left(\dot{J}_{2} / J_{2}\right) t \Delta \psi_{\mathrm{IAU} 2000}, \\
\mathrm{~d} \varepsilon_{\mathrm{J} 2 \mathrm{~d}} & =\left(\dot{J}_{2} / J_{2}\right) t \Delta \varepsilon_{\mathrm{IAU} 2000} .
\end{aligned}
$$

The second effect, which does not exist in the the $X, Y$ case, results from the fact that the $\mathrm{P} 03$ obliquity is different from the IAU 1980 obliquity that was used when estimating the IAU 2000A nutation amplitudes. To compensate for this change, it is necessary to multiply the amplitudes of the nutation in longitude by $\sin \epsilon_{\mathrm{IAU} 2000} / \sin \epsilon_{\mathrm{P} 03}=1.000000470$.

The correction for terms larger than $1 \mu$ as to be added to the IAU 2000A nutation in longitude for this effect is, in $\mu$ as:

$\mathrm{d}_{\epsilon} \psi=-8.1 \sin \Omega-0.6 \sin 2(F-D+\Omega)$, which can also be written as functions of $\Delta \psi_{\text {IAU2000 }}$ and $\Delta \varepsilon_{\text {IAU2000, }}$, the IAU 2000A nutation angles in longitude and obliquity respectively:

$\mathrm{d}_{1} \psi=\left[\left(\sin \epsilon_{\mathrm{IAU} 2000} / \sin \epsilon_{P 03}\right)-1\right] \Delta \psi_{\mathrm{IAU} 2000}$.

The accuracy in the CIP location is limited by the uncertainties in the parameters of the precession model appearing in Eq. (40) (i.e. the frame biases, precession rates and $J_{2}$ rate value) and of the nutation amplitudes. Realistic uncertainties for those parameters are (according to $\mathrm{C} 05$ ) of the order of $150 \mu$ as for the frame biases, $150 \mu$ as/cy for the linear term in $X$ and $Y$ (or equivalently $400 \mu \mathrm{as} / \mathrm{cy}$ and $150 \mu \mathrm{as} / \mathrm{cy}$ for the precession rates in longitude and obliquity, respectively) and $2 \mathrm{mas} / \mathrm{cy}^{2}$ for the $J_{2}$ rate; according to Mathews et al. (2002), there is an uncertainty of the order of $50 \mu$ as in the amplitude of the largest nutation term (i.e. the 18.6-yr term) and the free core nutation is not part of the IAU 2000A nutation model. The accuracy that can really be achieved for predicting the CIP location is therefore of the order of 2 mas after one century, even though the model itself, for any given values of the precession-nutation parameters, is of microarcsecond precision.

\subsubsection{Dependence on the choice of method}

The differences between the various methods for computing the position of the CIP are, as described in Sects. 3.3.1 and 3.3.2, in the way (i) the bias contribution and (ii) the precession-nutation contribution are treated. The comparison of the semi-analytical expressions obtained for $X, Y$ by three different methods agree to better than $1 \mu$ as after one century, which shows that the predicted discrepancies in the computed CIP position resulting from the choice of the method are below $1 \mu$ as after a century. These comparisons are based upon the $\mathrm{P} 03$ precession model and the IAU 2000A nutation model (including frame biases) with the slight modifications for conformity with the P03 precession that were described earlier.

The P03 series developments for $X$ and $Y$ described in $\mathrm{C} 03 \mathrm{c}$ were obtained by using the Sect. 3.1 method. They are provided in the form of Eqs. (7) and (8), the polynomial parts of which can be written as:

$$
\begin{aligned}
X_{\mathrm{P} 03}(\text { polyn })= & -0 \text { o. }^{\prime} 016617+2004{ }^{\prime \prime} 191898 t-0{ }^{\prime} .4297829 t^{2} \\
& -0{ }^{\prime} 19861834 t^{3}+0 .^{\prime} 000007578 t^{4}
\end{aligned}
$$

$$
\begin{aligned}
Y_{\mathrm{P} 03}(\text { polyn })= & -0 .^{\prime} 006951-0 .^{\prime} 025896 t-22^{\prime \prime} 4072747 t^{2} \\
& +0 .^{\prime} 00190059 t^{3}+0 .{ }^{\prime} 001112526 t^{4} \\
& +0 .^{\prime} 0000001358 t^{5}
\end{aligned}
$$

The polynomial parts of the P03 series developments for $X, Y$ derived from (3.2.2) can be written as:

$$
\begin{aligned}
X_{\mathrm{P} 03}^{\prime}(\text { polyn })= & -0 !^{\prime} 016617+2004{ }^{\prime \prime} 191898 t-0{ }^{\prime} 4297821 t^{2} \\
& -0 !^{\prime} 19861834 t^{3}+0{ }^{\prime} 000007578 t^{4}
\end{aligned}
$$

$$
\begin{aligned}
Y_{\mathrm{P} 03}^{\prime}(\text { polyn })= & -0 .^{\prime} 006951-0.025896 t-22^{\prime \prime} .4072744 t^{2} \\
& +0 .^{\prime} 00190059 t^{3}+0 .{ }^{\prime} 001112526 t^{4} \\
& +0 .^{\prime} 0000001358 t^{5}
\end{aligned}
$$


As already mentioned in Sect. 2.1, the polynomial parts of the $X, Y$ series originate from precession, except for the contribution from the frame bias (see Sect. 3.3.1) and from cross nutation terms. The latter contribute as a constant term in $Y_{P 03}$ (both Eqs. (49) and (51)) of $132 \mu$ as and a secular term in $X_{P 03}$ (both Eqs. (48) and (50)) of $4 \mu$ as per century.

\subsubsection{Application to pre-2003 VLBI EOP time series}

The identification between rotation matrix components and the CIP (Eq. (23)) suggests a convenient way of transforming existing VLBI Earth orientation parameter (EOP) time series to refer to the new precession-nutation models. Included in the EOP time series are the observed coordinates of the pole, expressed as "nutation offsets", corrections $\delta \psi, \delta \epsilon$ with respect to the IAU 1976/1980 precession-nutation models. In 2003, the reference model was changed to IAU 2000A, as set out in IERS Conventions (2003), with the observed pole offsets presented not as nutation components but instead directly as corrections $\delta X, \delta Y$ to the pole vector. When comparing EOP records from before 2003 with more recent results, it is necessary to transform the tabulated nutation offsets $\delta \psi_{1980}, \delta \epsilon_{1980}$ into the corresponding (and much smaller) $\delta X_{2000}, \delta Y_{2000}$.

The nutation offsets encompass both the unpredictable variations in Earth attitude that arise from geophysical causes but also the shortcomings of the IAU 1976/80 precessionnutation models. The latter, in addition to a multitude of comparatively small periodic terms, contain large fixed and secular components arising from frame bias and precessionrate errors respectively. In C03a it was shown that the pre-2003 practice of applying the frame bias and precession corrections as if they were nutation, i.e. applying them with respect to the mean equator and equinox of date rather than with respect to the GCRS and mean equator and equinox of J2000, respectively, introduced cross terms of significant size, of the order of 1 mas after 1 century. It is important that this is correctly handled in the $\delta \psi, \delta \epsilon$ to $\delta X, \delta Y$ transformation. This can be done by exploiting Eq. (23) to extract the CIP $X, Y$ from the IAU 1976/80-based rotation matrix and then to compare it with the CIP obtained by the current reference model, IAU 2000A.

The procedure is as follows:

1. Evaluate the IAU 1980 nutation components of date, $\Delta \psi_{1980}, \Delta \epsilon_{1980}$.

2. Add the EOP values $\delta \psi, \delta \epsilon$ to give the VLBI-observed nutation with respect to the IAU 1976 precession, $\left(\Delta \psi_{\mathrm{obs}}, \Delta \epsilon_{\mathrm{obs}}\right)_{1976}$.

3. Compute the IAU 1980 obliquity of date.

4. Using the results from steps 2 and 3, form the observed nutation matrix.

5. Compute the IAU 1976 precession matrix.

6. Combine the matrices from steps 4 and 5 to form the observed NPB matrix $\mathbf{M}_{\mathrm{obs}}$; elements $(3,1)$ and $(3,2)$ of this matrix are the GCRS $X, Y$ coordinates of the observed CIP.

7. Compute the IAU 2000A NPB matrix $\mathbf{M}_{2000 \mathrm{~A}}$; elements $(3,1)$ and $(3,2)$ are the GCRS $X, Y$ coordinates of the IAU $2000 \mathrm{~A}$ CIP.
8. Difference the two CIPs from steps 6 and 7 to give the observed corrections to the IAU 2000A precession-nutation:

$$
\begin{aligned}
\delta X_{2000 \mathrm{~A}} & =\mathbf{M}_{\mathrm{obs}}(3,1)-\mathbf{M}_{2000 \mathrm{~A}}(3,1), \\
\delta Y_{2000 \mathrm{~A}} & =\mathbf{M}_{\mathrm{obs}}(3,2)-\mathbf{M}_{2000 \mathrm{~A}}(3,2) .
\end{aligned}
$$

Equation (52) is a rigorous procedure for transforming existing VLBI nutation offsets with respect to the IAU 1976-1980 precession-nutation into corrections to the IAU 2000A CIP GCRS $X, Y$ coordinates. Whereas simpler formulations exist for transforming nutation offsets using pre-2003 practices (e.g. Vondrák et al. 2003), locating the CIP in the GCRS to microarcsecond accuracy requires a rigorous procedure such as that just described.

\section{The position of the $\mathrm{ClO}$}

Here we compare different possible ways of computing the GCRS position of the celestial intermediate origin.

The expressions for the various quantities have been developed into series by using the software package GREGOIRE. The polynomial parts of these series are provided in the following sections, and the number of periodic terms are listed in Tables 1 and 2. The complete series are available as tables in electronic form (see Appendix A). The various significant points on the CIP equator, and their relationship to the Earth rotation angle and the equation of the origins, are shown in Fig. 1.

\subsection{Method using the CIO locator s}

The GCRS position of the CIO derived from the kinematical definition of the CIO depends on an integral that involves the motion of the CIP.

Table 1. Comparison between various series positioning the CIO, $0.1 \mu$ as resolution.

\begin{tabular}{cccccc}
\hline \hline Quantity & \multicolumn{6}{c}{ Number of periodic terms in } \\
& $t^{0}$ & $t$ & $t^{2}$ & $t^{3}$ & $t^{4}$ \\
\hline$s$ & 24 & 125 & 21 & 2 & \\
$s+X Y / 2$ & 33 & 3 & 25 & 4 & 1 \\
$s+X Y / 2+D$ & 33 & 3 & 1 & 1 & \\
$\mathrm{EO}+\Delta \psi \cos \epsilon_{A}$ & 33 & 1 & & & \\
$y_{\text {CIO }}$ & 33 & 3 & 25 & 4 & 1 \\
\hline
\end{tabular}

Table 2. Size of the $x_{\mathrm{CIO}}, y_{\mathrm{CIO}}, z_{\mathrm{CIO}}$ series of periodic terms. See Sect. 4.3.

\begin{tabular}{clllll}
\hline \hline Coordinate & $t^{0}$ & $t$ & $t^{2}$ & $t^{3}$ & $t^{4}$ \\
\hline$x$ & 27 & 196 & 28 & 5 & 1 \\
$y$ & 33 & 3 & 25 & 4 & 1 \\
$z$ & 1306 & 254 & 36 & 4 & 1 \\
\hline
\end{tabular}




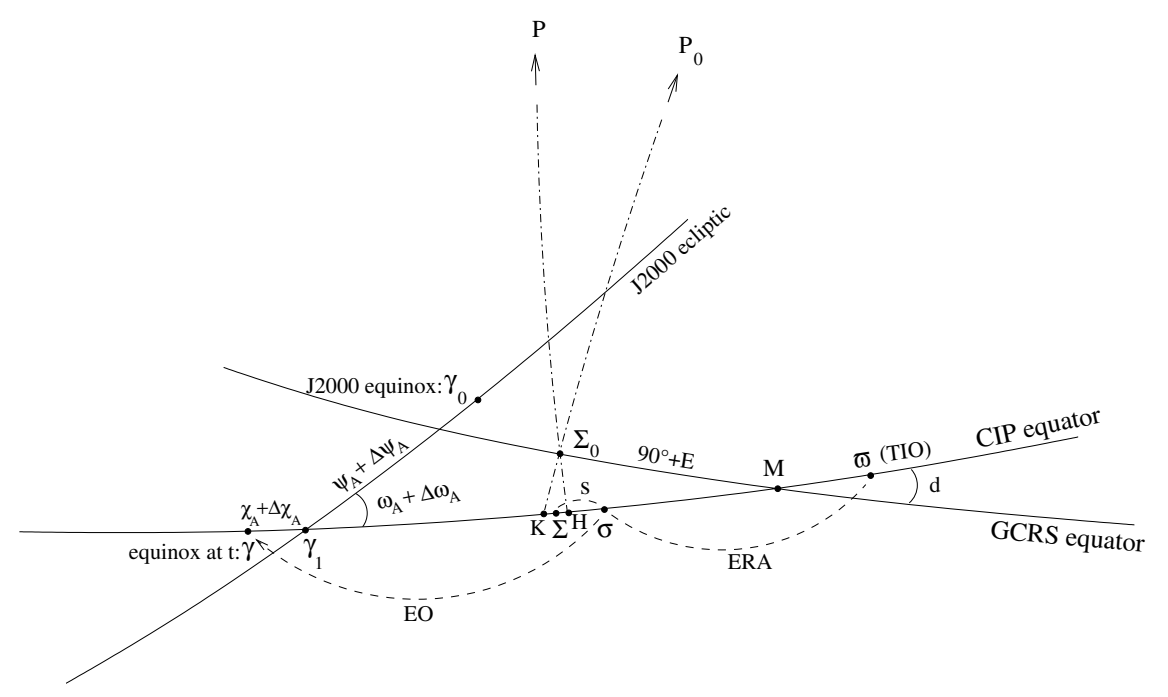

Fig. 1. The relationship between the CIO, the equinox and other significant points: $\Sigma_{0}$ is the GCRS origin, $\mathrm{M}$ is the ascending node of the CIP equator on the GCRS equator, $\Sigma$ is the point on the CIP equator such that $\widehat{\Sigma M}=\widehat{\Sigma_{0} M}, \gamma_{0}$ is the J2000 equinox, $\gamma$ is the equinox of date and $\gamma_{1}$ is the ascending node of the J2000 ecliptic on the CIP equator. $\mathrm{K}$ and $\mathrm{H}$ are the intersections of the GCRS meridian and the CIP meridian coming through $\Sigma_{0}$, respectively with the CIP equator. $\varpi$ is the TIO, ERA $=\widehat{\sigma \varpi}$ is the Earth rotation angle $(\mathrm{ERA})$ and EO $=\widehat{\sigma \gamma}$ is the equation of the origins.

The expression for the quantity $s$, called the "CIO locator", as a function of the CIP coordinates $X, Y, Z$, is:

$s(t)=\int_{t_{0}}^{t} \frac{[X(t) \dot{Y}(t)-\dot{X}(t) Y(t)]}{1+Z} \mathrm{~d} t-C_{0}$,

The expression for $s$ can be obtained either directly by computing the above integral, or through the computation of other quantities that can be expressed in fewer terms.

\subsubsection{Direct computation of $s$}

Equation (53) was used by Capitaine et al. (1986) to provide a semi-analytical expression consistent with the IAU1977/1980 precession-nutation model, including a polynomial in $t$ and a trigonometric part depending on the literal fundamental arguments of nutation, that allows the GCRS position of the CIO to be predicted directly as a function of time.

The semi-analytical expression for $s$ has the following form:

$$
\begin{aligned}
s(t)= & C_{0}-X_{0} Y_{0} / 2+\sum_{i} s_{i} t^{i} \\
& +\sum_{k}\left[\left(C_{s, 0}\right)_{k} \sin \alpha_{k}+\left(C_{c, 0}\right)_{k} \cos \alpha_{k}\right] \\
& +\sum_{k, j}\left[\left(C_{s, j}\right)_{k} \sin \alpha_{k}+\left(C_{c, j}\right)_{k} \cos \alpha_{k}\right] t^{j} .
\end{aligned}
$$

The computation of Eq. (53) based on the P03/IAU-2000Abased expressions for the coordinates $X$ and $Y$ as functions of time produced the following expression for the polynomial part of $s$ with a cut-off of $0.1 \mu$ as:

$$
\begin{aligned}
s_{\mathrm{P} 03}= & 94.0+3825.39 t+1.21 t^{2} \\
& +36287.09 t^{3}-4.61 t^{4}-0.57 t^{5} .
\end{aligned}
$$

The periodic part of this P03/IAU 2000 expression for $s$ with a cut-off of $0.1 \mu$ as includes 24 Fourier terms and 125, 21

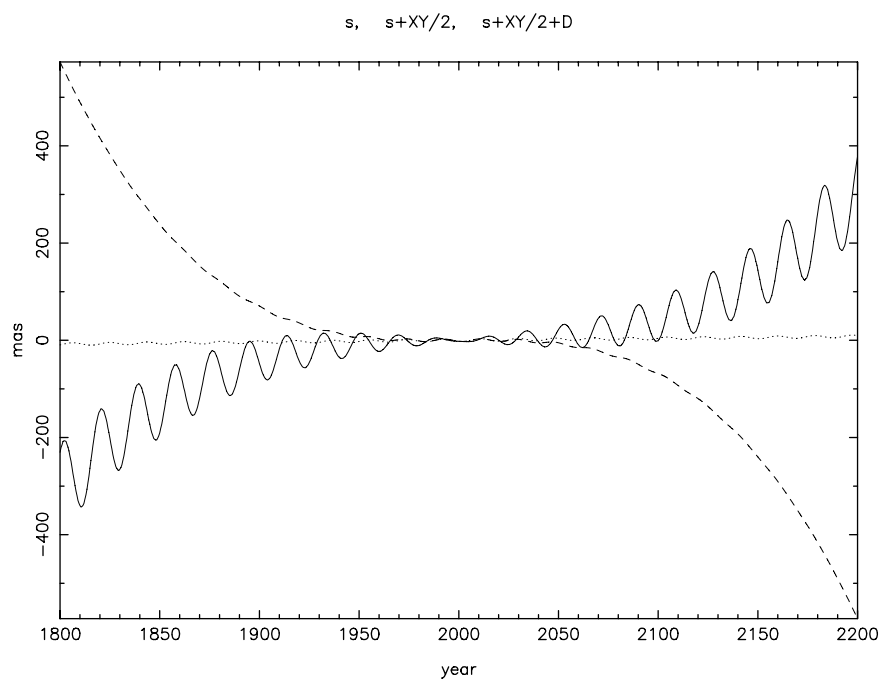

Fig. 2. Three different quantities from which $s$ can be obtained, thereby locating the CIO. The solid line is $s$ itself. The function $s+X Y / 2$ (dashed line) eliminates most of the nutation and hence reduces the number of terms needed in a series representation. The function $s+X Y / 2+D$ (the almost horizontal dotted line) is smoother still. A fourth quantity that can be used to locate the CIO is the equation of the origins (EO: see Sect. 4.2). The EO is not shown because it is dominated by precession in right ascension and hence changes by over $5^{\circ}$ in the 400 -year interval depicted.

and 2 Poisson terms of degree 1, 2 and 3, respectively (see Table 1).

The quantity $s$ is the solid line in Fig. 2.

\subsubsection{Expression for the quantity $s+X Y / 2$}

For recent models (see for example C03b and IERS Conventions 2003), the numerical development used to determine the GCRS position of the CIO gives not $s$ itself but instead 
the quantity $s+X Y / 2$, which requires fewer terms to reach the same accuracy.

This quantity is defined by:

$s+\frac{X Y}{2}=\int_{t_{0}}^{t} \dot{X(t)} Y(t) \mathrm{d} t-C_{0}+\delta s$,

where $\delta s$ is such that:

$\delta s=-\int_{t_{0}}^{t}(2 a-1) \frac{X \dot{Y}-Y \dot{X}}{2} \mathrm{~d} t$,

$a$ being given by (5).

Equation (56) provides an expression for $s$ which is rigorously equivalent to Eq. (53) and $\delta s$ is a small quantity of the order of $1 \mu$ as after a century.

The expression for $s+X Y / 2$ has the following form:

$$
\begin{aligned}
s(t)+X Y / 2= & C_{0}+\sum_{i} s_{i} t^{i} \\
& +\sum_{k}\left[\left(C_{s, 0}\right)_{k} \sin \alpha_{k}+\left(C_{c, 0}\right)_{k} \cos \alpha_{k}\right] \\
& +\sum_{k, j}\left[\left(C_{s, j}\right)_{k} \sin \alpha_{k}+\left(C_{c, j}\right)_{k} \cos \alpha_{k}\right] t^{j},
\end{aligned}
$$

the numerical value for $C_{0}$ being fixed by the condition that UT1 be continuous through the transition on 2003 January 1.0 from the classical procedure based on the previous definition of UT1 to the new procedure based on the IAU 2000 definition.

The computation based on the P03/IAU-2000A-based expressions for the coordinates $X$ and $Y$ as functions of time produced the following expression for the polynomial part of $s+X Y / 2$, using a cut-off of $0.1 \mu$ as $(\mathrm{C} 03 \mathrm{~b})$ :

$$
\begin{aligned}
(s+X Y / 2)_{\mathrm{P} 03}= & 94.0+3808.65 t-122.68 t^{2} \\
& -72574.11 t^{3}+27.98 t^{4}+15.62 t^{5} .
\end{aligned}
$$

The series development for the contribution of Eqs. (57) to (59) is:

$0.5 t^{5}+0.1 t^{3}-0.1 t^{2}-1.1 t^{3} \cos \Omega-0.1 t^{3} \cos 2 \odot-0.1 t^{2} \sin \Omega$.

The periodic part of $s+X Y / 2$, again with a cut-off of $0.1 \mu$ as, includes 33 Fourier terms and 3, 25, 2 and 1 Poisson terms of degree 1, 2, 3 and 4, respectively (see Table 1).

The function $s+X Y / 2$ is the dashed line in Fig. 2. Compared with $s$ itself, it shows greatly reduced nutation effects and is accordingly simpler to model to a given precision.

\subsubsection{Expression for the quantity $s+X Y / 2+D$}

While the expressions for the quantities $s$ and $s+X Y / 2$ include non-negligible $t^{3}$ terms plus a number of Poisson terms of degree 2, no such terms are present in the expression for the quantity $\mathrm{EO}+\Delta \psi \cos \epsilon_{A}$ (cf. Sect. 4.2). This indicates that these terms can be eliminated when locating the CIO with respect to an appropriate reference point on the CIP equator. We have therefore looked for a quantity from which $s$ can be obtained as conveniently as it can from $s+X Y / 2$ but which has as concise a model as that for the EO.
The following expression has been tried, based on theoretical reasons that will be explained in Sect. 4.7.1:

$s+X Y / 2+D=s+X Y / 2-Y_{2} t^{2}\left[\left(X_{1} / 3\right) t+X_{\text {nut }}\right]$,

where $X_{1}$ and $Y_{2}$ are the linear and the quadratic terms in the polynomial part of $X$ and $Y$ in Eqs. (7) and (8), respectively and $X_{\text {nut }}$ is the periodic part of the $X$ expression.

The computation based on the P03/IAU-2000A-based expressions for the coordinates $X$ and $Y$ as functions of time produced the following expression for the polynomial part of $s+X Y / 2+D$, with a cut-off of $0.1 \mu$ as:

$$
\begin{aligned}
(s+X Y / 2+D)_{\mathrm{P} 03}= & 94.0+3808.65 t-122.68 t^{2} \\
& +27.98 t^{4}+15.62 t^{5} .
\end{aligned}
$$

The periodic part, with a cut-off of $0.1 \mu$ as, includes 33 Fourier terms and 3, 1 and 1 Poisson terms of degree 1, 2 and 3, respectively (see Table 1).

The function $s+X Y / 2+D$ is the dotted line in Fig. 2 .

\subsection{Method using the "equation of the origins"}

Greenwich sidereal time at date $t$ (GST, or $\theta)$ is related to ERA by subtracting the "equation of the origins", denoted EO (i.e. $\mathrm{GST}=\theta-\mathrm{EO}$ ). In other words, the EO is the CIO right ascension, or equivalently the "intermediate right ascension" of the equinox (according to the NFA IAU Working Group Recommendations on terminology; see Capitaine et al. 2006): EO is the angular distance between the CIO and the equinox along the CIP equator, reckoned westwards from the CIO. The EO's role as the link between the classical and CIO based methods is illustrated by the following expressions for the GCRSto-TIRS rotation matrix $\mathbf{R}$ :

$$
\begin{aligned}
\mathbf{R}(\mathrm{TT}, \mathrm{UT}) & =R_{3}(\mathrm{ERA}) \cdot \mathbf{M}_{\mathrm{CIO}} \\
& =R_{3}(\mathrm{ERA}) \cdot R_{3}(-\mathrm{EO}) \cdot \mathbf{M}_{\text {class }} \\
& =R_{3}(\mathrm{GST}) \cdot \mathbf{M}_{\text {class }} .
\end{aligned}
$$

The three options correspond to (i) generating the CIO based GCRS to CIRS matrix directly, ready to be used in conjunction with the Earth rotation angle; (ii) using the EO to transform the classical matrix into the CIO based matrix; and (iii) using the EO to convert ERA to GST.

The theoretical expression for GST $=\widehat{\Upsilon \varpi}$ as a function of time is the following (cf. C03b):

$\mathrm{GST}=\theta+\int_{0}^{t}\left(\psi_{A} \dot{\overrightarrow{+\Delta}} \psi_{1}\right) \cos \left(\omega_{A}+\Delta \epsilon_{1}\right) \mathrm{d} t-\left(\chi_{A}+\Delta \chi_{A}\right)$,

where $\psi_{A}$ and $\omega_{A}$ are the precession quantities in longitude and obliquity referred to the $\mathrm{J} 2000$ ecliptic, $\Delta \psi_{1}$ and $\Delta \epsilon_{1}$ are the nutation quantities referred to the J2000 ecliptic and the quantity $\chi_{A}+\Delta \chi_{A}$ is the distance along the CIP equator between the equinox $\Upsilon$ and the intersection $\Upsilon_{1}$ of the J2000 ecliptic (i.e. it is the accumulated precession of the ecliptic along the CIP equator).

The development for GST includes a polynomial in $t$ (i.e. Greenwich mean sidereal time, GMST), plus the "complete equation of the equinoxes" that includes the classical "equation of the equinoxes", $\Delta \psi \cos \epsilon_{A}$, plus a complementary 
part, denoted $\mathrm{dEE}$, that comes from the coupling between precession and nutation.

The expression for the quantity EO can be derived from Eq. (63). Using the relationship:

$\Delta \chi_{A}=-\Delta \psi \cos \epsilon_{A}+\Delta \psi_{1} \cos \omega_{A}+\cdots$,

which corresponds to approximation at the level of $0.05 \mu \mathrm{as} / \mathrm{cy}$, EO can be written as (see Aoki \& Kinoshita 1983; Capitaine \& Gontier 1993; and C03b):

$$
\begin{aligned}
\mathrm{EO}=\theta-\mathrm{GST}= & -\int_{0}^{t}\left(\psi_{A} \dot{\overrightarrow{+} \Delta} \psi_{1}\right) \cos \left(\omega_{A}+\Delta \epsilon_{1}\right) \mathrm{d} t \\
& +\chi_{A}-\Delta \psi \cos \epsilon_{A}+\Delta \psi_{1} \cos \omega_{A},
\end{aligned}
$$

or equivalently:

$$
\begin{gathered}
\mathrm{EO}=-\int_{0}^{t} \dot{\psi_{A}} \cos \omega_{A} \mathrm{~d} t+\chi_{A}-\Delta \psi \cos \epsilon_{A} \\
-\mathrm{dEE}_{\mathrm{S}}-\mathrm{dEE}_{\mathrm{p}},
\end{gathered}
$$

where $\mathrm{dEE}_{\mathrm{s}}$ and $\mathrm{dEE}_{\mathrm{p}}$ are the periodic and secular components of the "complementary part", dEE, of the equation of the equinoxes, which can be written at the $\mu$ as level (see Aoki \& Kinoshita 1983; and C03b), using the same notations as in Eq. (63):

$$
\begin{aligned}
\mathrm{dEE}_{\mathrm{S}}= & {\left[\int_{0}^{t} \dot{\Delta \psi_{1}} \Delta \epsilon_{1} \sin \omega_{A} \mathrm{~d} t\right]_{\mathrm{s}} } \\
& +\frac{1}{2}\left[\int_{0}^{t} \dot{\psi_{A}} \Delta \epsilon_{1}^{2} \cos \omega_{A} \mathrm{~d} t\right]_{\mathrm{S}} \\
\mathrm{dEE}_{\mathrm{p}}= & +\int_{0}^{t} \dot{\psi_{A}} \sin \omega_{A} \Delta \epsilon_{1} \mathrm{~d} t \\
& +\int_{0}^{t} \dot{\widehat{\Delta \psi_{1}}}\left(\omega_{A}-\epsilon_{0}\right) \sin \omega_{A} \mathrm{~d} t \\
& +\int_{0}^{t}\left[\dot{\widehat{\Delta \psi_{1}}} \Delta \epsilon_{1} \sin \omega_{A} \mathrm{~d} t\right]_{\mathrm{p}} \\
& +\frac{1}{2}\left[\int_{0}^{t} \dot{\psi_{A}} \Delta \epsilon_{1}^{2} \cos \omega_{A} \mathrm{~d} t\right]_{\mathrm{p}} .
\end{aligned}
$$

The expression for the quantity EO can be provided in a semianalytical form similar to that of the quantities $s$ and $s+X Y / 2$ by a semi-analytical computation of Eq. (65). Note that the expression for $-\mathrm{dEE}_{\mathrm{s}}$, which is is in fact included in the polynomial part of GST, is equal to the secular contribution of nutation to the quantity $s$. The expression for $-\mathrm{dEE}_{\mathrm{p}}$ is a series of mainly Fourier terms similar to those appearing in the quantity $s+X Y / 2$.

The expression for EO based on the P03/IAU-2000A-based expressions for the precession-nutation quantities is, with a cutoff of $0.1 \mu$ as $t$, for the amplitudes of the periodic terms:

$$
\begin{aligned}
\mathrm{EO}_{\mathrm{P} 03}= & -0 .{ }^{\prime} 014506-4612^{\prime \prime} 15653353 t \\
& -1^{\prime \prime} 39158165 t^{2}+0 .{ }^{\prime} 00000044 t^{3} \\
& +0 .{ }^{\prime} 000029956 t^{4} \\
& -\Delta \psi \cos \epsilon_{A}+\sum_{k}\left[\left(C_{s, 0}^{\prime}\right)_{k} \sin \alpha_{k}+\left(C_{c, 0}^{\prime}\right)_{k} \cos \alpha_{k}\right] \\
& +0 .{ }^{\prime} 00000087 t \sin \Omega .
\end{aligned}
$$

Note that, in this computation, the nutation angles $\Delta \psi_{1}$ and $\Delta \epsilon_{1}$ have been derived from the nutation angles $\Delta \psi$ and $\Delta \epsilon$ referred to the ecliptic of date through Eq. (35) with the already mentioned approximation (i.e. $1 \mu$ as after two centuries in EO).

With a cut-off of $0.1 \mu \mathrm{as}$, the periodic part of Eq. (69) includes, additionally to the classical nutation in right ascension, 33 Fourier terms and 1 Poisson term of degree 1, which corresponds to the development of $\mathrm{dEE}_{\mathrm{p}}$. The expression for $\mathrm{dEE}_{\mathrm{p}}$ has, for a given accuracy, fewer terms than that for the other quantities considered in the previous sections (see Table 1). This can be explained through the relationships between EO and those quantities (see Sect. 4.7).

\subsection{Expressions for the GCRS CIO coordinates based on the $M_{\mathrm{CIO}}$ matrix elements}

The position of the CIO can alternatively be provided by its GCRS coordinates, which form the top row of the $\mathbf{M}_{\mathrm{CIO}}$ matrix. They can be written, by substituting $\beta=s$ into Eq. (25), as:

$\mathbf{M}(1,1)=x_{\mathrm{CIO}}=\left(1-a X^{2}\right) \cos s+a X Y \sin s$,

$\mathbf{M}(1,2)=y_{\mathrm{CIO}}=-a X Y \cos s-\left(1-a Y^{2}\right) \sin s$,

$\mathbf{M}(1,3)=z_{\mathrm{CIO}}=-X \cos s+Y \sin s$,

where $a=1 /(1+Z)$, and $X, Y, Z$ are the GCRS coordinates of the CIP.

We have computed semi-analytical series for these expressions. Each series has six polynomial coefficients, plus a sum of periodic terms, the number of which is shown in Table 2. We note that the $y_{\mathrm{CIO}}$ series is similar to that of $-(s+X Y / 2)$ (see Eq. (58)) and $z_{\mathrm{CIO}}$ is similar to $-X_{\mathrm{CIP}}$ (see Eq. (48)); in fact, the $x_{\mathrm{CIO}}$ series can be more simply computed through $\left(1-\left(y_{\mathrm{CIO}}^{2}+z_{\mathrm{CIO}}^{2}\right)\right)^{1 / 2}$.

The only series out of the three that could have a practical role is that for $y_{\text {CIO }}$ (see Table 1). The series for $z_{\text {CIO }}$ is much longer than the other two, being essentially the same as for the CIP $X$.

\subsection{Expressions for the GCRS CIO coordinates based on the equinox based matrix elements}

The GCRS CIO coordinates can also be derived from the elements of the $\mathbf{M}_{\text {class }}$ matrix and the EO, through Eq. (10).

Using the form Eq. (11) of the NPB matrix would provide expressions for $x_{\mathrm{CIO}}, y_{\mathrm{CIO}}, z_{\mathrm{CIO}}$ as functions of the classical precession quantities, $\psi_{A}, \omega_{A}$ and $\chi_{A}$, nutation quantities $\Delta \psi$ and $\Delta \epsilon$, the $\mathbf{J} 2000$ obliquity of the ecliptic $\epsilon_{0}$ and the frame biases $\xi_{0}, \eta_{0}$ and $\mathrm{d} \alpha_{0}$. However, as we already noted in Sect. 3.2.1, expressions for the elements derived from this form of the $\mathbf{M}_{\text {class }}$ matrix are not straightforward and using another form of the NPB matrix is more appropriate.

As for the CIP case, elements of the $\mathbf{M}_{\mathrm{FW}}$ form (cf. Eq. (15)) can provide simple expressions for the GCRS CIO coordinates as functions of the new precessionnutation-bias quantities referred to the ecliptic of date. 
These can be written as:

$$
\begin{aligned}
& x_{\mathrm{CIO}}=\mathbf{M}(1,1) \cos \mathrm{EO}-\mathbf{M}(2,1) \sin \mathrm{EO}, \\
& y_{\mathrm{CIO}}=\mathbf{M}(1,2) \cos \mathrm{EO}-\mathbf{M}(2,2) \sin \mathrm{EO}, \\
& z_{\mathrm{CIO}}=\mathbf{M}(1,3) \cos \mathrm{EO}-\mathbf{M}(2,3) \sin \mathrm{EO}
\end{aligned}
$$

with:

$$
\begin{aligned}
\mathbf{M}(1,1)= & \cos \psi^{\prime} \cos \gamma^{\prime}+\sin \psi^{\prime} \cos \phi^{\prime} \sin \gamma^{\prime}, \\
\mathbf{M}(1,2)= & \cos \psi^{\prime} \sin \gamma^{\prime}-\sin \psi^{\prime} \cos \phi^{\prime} \cos \gamma^{\prime}, \\
\mathbf{M}(1,3)= & -\sin \psi^{\prime} \sin \phi^{\prime}, \\
\mathbf{M}(2,1)= & \cos \epsilon^{\prime} \sin \psi^{\prime} \cos \gamma^{\prime} \\
& -\left(\cos \epsilon^{\prime} \cos \psi^{\prime} \cos \phi^{\prime}+\sin \epsilon^{\prime} \sin \phi^{\prime}\right) \sin \gamma^{\prime}, \\
\mathbf{M}(2,2)= & \cos \epsilon^{\prime} \sin \psi^{\prime} \sin \gamma^{\prime} \\
& +\left(\cos \epsilon^{\prime} \cos \psi^{\prime} \cos \phi^{\prime}+\sin \epsilon^{\prime} \sin \phi^{\prime}\right) \cos \gamma^{\prime}, \\
\mathbf{M}(2,3)= & \cos \epsilon^{\prime} \cos \psi^{\prime} \sin \phi^{\prime}-\sin \epsilon^{\prime} \cos \phi^{\prime}
\end{aligned}
$$

which allows us to compute semi-analytical series for $x_{\mathrm{CIO}}$, $y_{\mathrm{CIO}}, z_{\mathrm{CIO}}$ independently of those computed in Sect. 4.3.

\subsection{Expression relating $s, E O$ and the matrix $M_{\text {class }}$}

Combining Eqs. (3) and (10) we have:

$\mathbf{M}_{\mathrm{CIO}}=R_{3}(-\mathrm{EO}) \cdot \mathbf{M}_{\mathrm{class}}=R_{3}(-s) \cdot \mathbf{M}_{\Sigma}$

and hence:

$R_{3}(-s)=R_{3}(-\mathrm{EO}) \cdot \mathbf{M}_{\mathrm{class}} \cdot \mathbf{M}_{\Sigma}^{T}$.

Element $(2,1)$ of the matrix $R_{3}(-s)$ is $\sin (s)$ or, without significant loss of accuracy, $s$. Therefore we can obtain $s$ simply by evaluating the corresponding element of the right-hand side of Eq. (74). Writing the top and middle rows of the matrix $\mathbf{M}_{\text {class }}$ as $\boldsymbol{\Upsilon}$ and $\mathbf{y}$ respectively, and the top row of the matrix $\mathbf{M}_{\Sigma}$ as $\boldsymbol{\Sigma}$, this matrix element can be expanded as:

$s \simeq p \sin \mathrm{EO}+q \cos \mathrm{EO}$,

where $p=\Upsilon \cdot \boldsymbol{\Sigma}$ and $q=\mathbf{y} \cdot \boldsymbol{\Sigma}$. We can also solve this for EO:

$\mathrm{EO} \simeq \arctan \frac{p s-q w}{q s+p w}$,

where $w=\left(p^{2}+q^{2}-s^{2}\right)^{1 / 2}$. From Eq. (4),

$\Sigma \equiv\left(1-a X^{2},-a X Y,-X\right)$,

where $a=1 /(1+Z)$ and the CIP coordinates $X, Y, Z$ are the bottom row of $\mathbf{M}_{\text {class. }}$ Therefore if we have $\mathbf{M}_{\text {class }}$ and the EO, we can use expression Eq. (75) to calculate $s$. Conversely, if we have $\mathbf{M}_{\text {class }}$ and $s$, we can use Eq. (76) to calculate the EO.

For applications where numerical consistency is paramount, obtaining the EO from $s$ is arguably more useful than the reverse, as it enables one of the series for $s$ to act as the basis of the EO prediction (and ultimately the computation of GST), and eliminates the need for the polynomial part of the EO to cancel precisely the rapid motion of the equinox obtained from the top row of $\mathbf{M}_{\text {class. }}$. This advantage is, however, at the expense of some additional computation.

\subsection{Method using the property of orthogonality of the instantaneous motion of the $\mathrm{ClO}$}

An alternative (that we will not consider in detail here) to the usual quantities used for positioning the CIO has been developed by Kaplan (2003). This method is based on numerically integrating a simple vector differential equation for the position of a non-rotating origin on its reference sphere. This scheme directly yields the GCRS right ascension and declination of the $\mathrm{CIO}$ as a function of time.

The computation of the GCRS position of the CIO results in describing a point on the moving equator whose instantaneous motion is always orthogonal to the equator. If $\boldsymbol{n}(t)$ is a unit vector toward the instantaneous pole and $\boldsymbol{x}(t)$ is a unit vector toward an instantaneous origin, the condition to be fulfilled is:

$\dot{x}(t)=-[(x(t) \cdot \dot{n}(t)] n(t)$.

The CIO position evaluated in this way is used to form the top row of the $\mathbf{M}_{\mathrm{CIO}}$ matrix, which offers an alternative to the numerical evaluation of Eq. (70).

\subsection{Relationships between the methods}

\subsubsection{Relationships between the various points on the CIP equator for positioning the $\mathrm{CIO}$}

The quantity $s$ is the distance on the CIP equator of the CIO from the point $\Sigma$, which is such that $\Sigma_{0} N=\Sigma N, \mathrm{~N}$ being the node of the CIP equator on the GCRS equator.

The point $\Sigma$ on the CIP equator is nearly at the middle of the intersections (i) of the GCRS zero-meridian (point K) and (ii) of the intermediate meridian passing through $\Sigma_{0}$ (point H) (see Fukushima 2001). It is important to note that the quantity $s+X Y / 2$ nearly represents (i.e. equals, up to the fourth order in $X$ and $Y$ ) the distance, on the CIP equator, of the $\mathrm{CIO}$ from the point $\mathrm{K}$ (i.e. $s+X Y / 2$ is the $\mathrm{CIO}$ right ascension of point $\mathrm{K}$ ).

We have noted that, at a $0.1 \mu$ as level, the addition of the quantity $D=-Y_{2} t^{2}\left[\left(X_{1} / 3\right) t+X_{\text {nut }}\right]$ to the quantity $s+X Y / 2$ eliminates the $t^{3}$ term and the Poisson terms of degree 2 in the resulting expression. This means that the quantity $s+X Y / 2+D$ corresponds to the angular distance of the CIO from a point on the CIP equator of which the motion with respect to $\Sigma$ is expressed by those terms.

The quantity EO is related to the CIO locator, $s$, by:

$$
\begin{aligned}
\mathrm{EO}=\widehat{\sigma \Upsilon} & =\left(\sigma N-\sigma_{0} N\right)-\left(\Upsilon N-\Upsilon_{0} N\right) \\
& =s-\left(\Upsilon N-\Upsilon_{0} N\right),
\end{aligned}
$$

where the quantities $\left(\Upsilon N-\Upsilon_{0} N\right)$ and $\left(\sigma N-\sigma_{0} N\right)$ both include effects that come from the changing distance of $N$ from the CIO. The quantity EO $\left(=s-\left[\Upsilon N-\Upsilon_{0} N\right]\right)$ accounts for the accumulated precession and nutation in right ascension from J2000 to the epoch $t$, reckoned from the CIO. The relationship between the periodic part, denoted $s_{\mathrm{p}}$, of the quantity $s$ and the component $-\mathrm{dEE}_{\mathrm{p}}$ of the EO can be derived by equating the rotation along the CIP equator from the node $N$ to the 
TIO in the equinox based and CIO based transformations, as follows, at the $\mu$ as level:

$$
\begin{aligned}
s_{\mathrm{p}}-\mathrm{dEE}_{\mathrm{p}}= & -\frac{1}{2} \Delta \psi_{1} \Delta \epsilon_{1} \sin \epsilon_{0}-\frac{1}{2} \psi_{A} \sin \epsilon_{0} \Delta \epsilon_{1} \\
& +\frac{1}{12}\left(\psi_{A}\right)^{3} \sin \epsilon_{0} \Delta \epsilon_{1} \\
& +\frac{1}{4} \sin ^{2} \epsilon_{0} \cos \epsilon_{0} \psi_{A} \Delta \psi_{1}^{2}+\frac{1}{4} \sin ^{2} \epsilon_{0} \cos \epsilon_{0} \psi_{A}^{2} \Delta \psi_{1} \\
& +\frac{1}{2}\left(\omega_{A}-\epsilon_{0}\right) \sin \epsilon_{0} \Delta \psi_{1} .
\end{aligned}
$$

The above expression, which is the periodic part of the effects appearing in $s$ that come from the changing distance of $N$ from the CIO, shows why the expression for the quantity $\mathrm{dEE}_{\mathrm{p}}$ is simpler than that for the quantity $s$.

This expression also makes clear the simplification (see Table 1) that is obtained by using the quantities $s+X Y / 2$ or $s+X Y / 2+D$ instead of $s$. This is because the periodic part of the quantities $-X Y / 2$ and $-X Y / 2-D$ are approximately equal to the first two lines and the first three lines of Eq. (79), respectively.

\subsubsection{Relationships between the GCRS $\mathrm{CIO}$ coordinates and the $\mathrm{CIO}$ locator}

The quantity $s$ is sufficiently small for us to write Eq. (70), to an accuracy of $10 \mu$ as after one century, as:

$$
\begin{aligned}
& x_{\mathrm{CIO}} \simeq 1-X^{2} / 2, \\
& y_{\mathrm{CIO}} \simeq-(s+X Y / 2), \\
& z_{\mathrm{CIO}} \simeq-X+s Y,
\end{aligned}
$$

which shows the similarity between (i) the $y_{\mathrm{CIO}}$ and $s+X Y / 2$ series and (ii) the $z_{\mathrm{CIO}}$ and $-X_{\mathrm{CIP}}$ series.

\subsection{Accuracy considerations}

\subsubsection{Dependence on the precession-nutation model}

The CIO location depends only to a very small extent on the precession-nutation model. Therefore, the accuracy that can really be achieved for predicting the location of the CIO is better than a few $\mu$ as after a century when the quantity $s$ is used.

In contrast, the expression for the equinox CIO right ascension that includes the accumulated precession and nutation in right ascension is directly dependent on that model, which limits the accuracy of the EO quantity to a few mas after one century.

For example, changing from the IAU 2000 precession solution to the P03 solution gives rise to small changes, of the order of $0.1 \mu$ as to a few $\mu$ as for a century, in a few terms in the polynomial part of the quantities $s, s+X Y / 2$ and $s+X Y / 2+D$ (the largest being of $-2.7 \mu$ as in the quadratic term, the other changes all being less than $0.5 \mu \mathrm{as}$ ). In contrast, this gives rise in the expression for EO to much larger changes, of the order of $\Delta \mathrm{EO}=358 t-5913 t^{2}+5 t^{3}+125 t^{4}$.

However, as the resulting uncertainties in the EO are in the equinox position rather than the $\mathrm{CIO}$ position, the $\mathrm{EO}$ can be used to locate the latter to a comparable accuracy to that from the other methods provided that formulations compatible at microarcsecond level be used for the EO and equinox based NPB.

\subsection{Dependence on the choice of method}

We have compared the series Eqs. (54), (58) and (61) that we obtained using the three different expressions (i.e. $s, s+$ $X Y / 2$ and $s+X Y / 2+D$ ) for the CIO locator described in Sects. 4.1.1-4.1.3, respectively, based on the P03/IAU2000A series that were described earlier for $X$ and $Y$.

We have also compared the series for the GCRS CIO coordinates that we obtained using the two different expressions for the elements of the GCRS to CIRS transformation matrix described in Sects. 4.3 and 4.4. Those expressions for $x_{\mathrm{CIO}}, y_{\mathrm{CIO}}, z_{\mathrm{CIO}}$ were based on the P03/IAU2000A series that were described earlier for the elements of the CIO based and equinox based NPB matrix, respectively, and for the quantities $s$ and $\mathrm{EO}$, respectively.

These various comparisons showed that the semi-analytical expressions for positioning the $\mathrm{CIO}$ all agree at the $\mu$ as level after one century.

\section{Numerical comparisons}

All the comparison tests reported in the following sections are based upon the P03 precession model and the IAU 2000A nutation model (including frame biases) with the slight modifications (Eqs. (44) and (46)) needed to make the nutation angles conform to the $\mathrm{P} 03$ precession.

\subsection{Semi-analytical expressions for $X$ and $Y$ against classical precession-nutation}

The differences between the predictions of CIP $X, Y$ from the semi-analytical series (Eqs. (50) and (51)) and those from evaluating the elements $(3,1)$ and $(3,2)$ of the classical NPB matrix are shown in Figs. 3 and 4. The matrix elements were generated using the Fukushima-Williams 4-rotation method (Eq. (38)).

\subsection{Semi-analytical expression for $s$ against numerical integration}

The differences between $s$ determined from the semi-analytical series for $s+X Y / 2$ (see Eq. (58)) and from direct numerical integration are shown in Fig. 5. The numerical integration was of Eq. (53).

\subsection{Semi-analytical expression for $s$ against numerical integration based on the kinematical definition of the $\mathrm{CIO}$}

An alternative numerical-integration method is to implement the CIO's kinematical definition literally, generating the GCRS-to-CIRS rotation matrix by successive infinitesimal rotations about an axis through the instantaneous line of nodes. For CIP velocity $(\dot{x}, \dot{y})$ in the instantaneous coordinate system 
CIP $X$ : series minus $F-W$

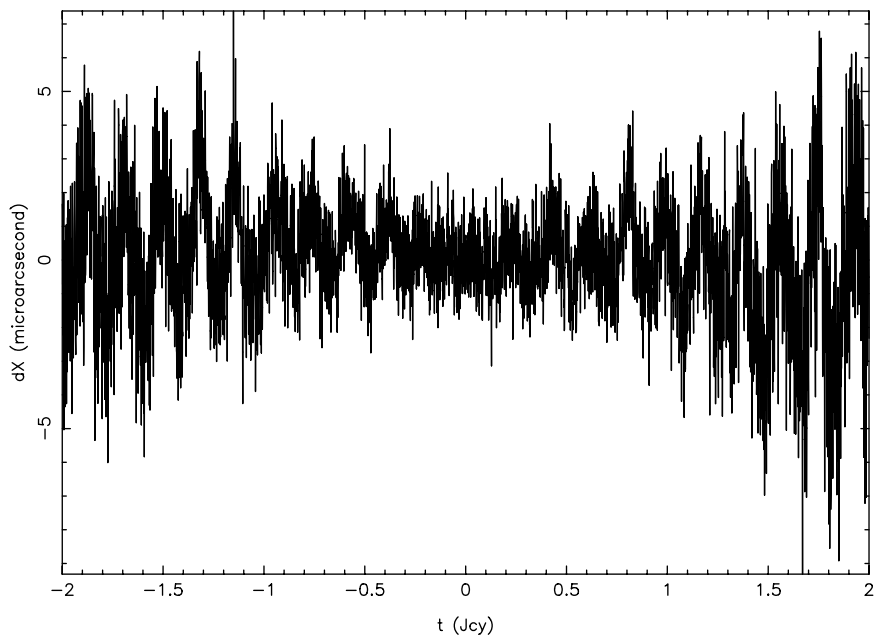

Fig. 3. Comparison between the CIP $X$-coordinate determined from the semi-analytical series and by evaluating the matrix element $(3,1)$ of the classical NPB matrix. See Sect. 5.1.

CIP Y: series minus $\mathrm{F}-\mathrm{W}$

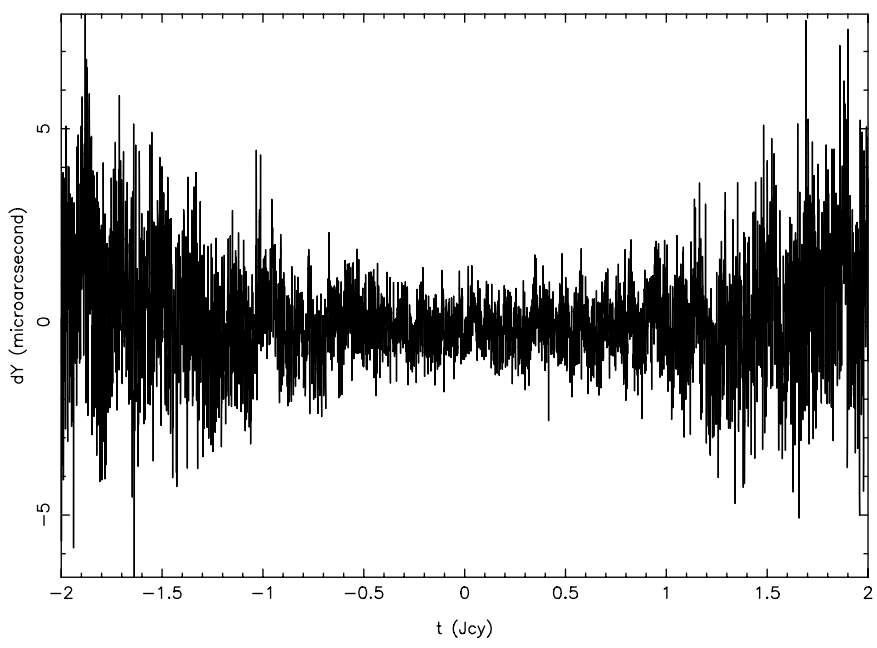

Fig. 4. Comparison between the CIP $Y$-coordinate determined from the semi-analytical series and by evaluating the matrix element $(3,2)$ of the classical NPB matrix. See Sect. 5.1.

and a time-step $\Delta t$, the CIP moves by $(\dot{x} \Delta t, \dot{y} \Delta t)$, or $(\Delta x, \Delta y)$. This can be represented as a "rotation vector" (see Sect. 2.3), the components of which are $(-\Delta y,+\Delta x, 0)$, the zero $z$ component being the NRO condition. The vector can be transformed into a rotation matrix by applying Eq. (18) for small values of $(\Delta x, \Delta y)$, leading to:

$\mathbf{M}(t) \simeq\left[\prod_{0}^{t}\left(\begin{array}{ccc}1 & -\Delta x \Delta y / 2 & -\Delta x \\ -\Delta x \Delta y / 2 & 1 & -\Delta y \\ +\Delta x & +\Delta y & 1\end{array}\right)\right] \mathbf{M}(0)$,

where $\mathbf{M}(0)$ is the GCRS to CIRS rotation matrix at epoch and $\mathbf{M}(t)$ the matrix at date $t$. (For sufficiently small time-steps, matrix elements $(1,2)$ and $(2,1)$ can be set to zero.)

Because $(\Delta x, \Delta y)$ are in the instantaneous coordinate system, i.e. the CIRS, the changes $(\Delta X, \Delta Y)$ in the GCRS position of the CIP must be rotated before use. We first complete the

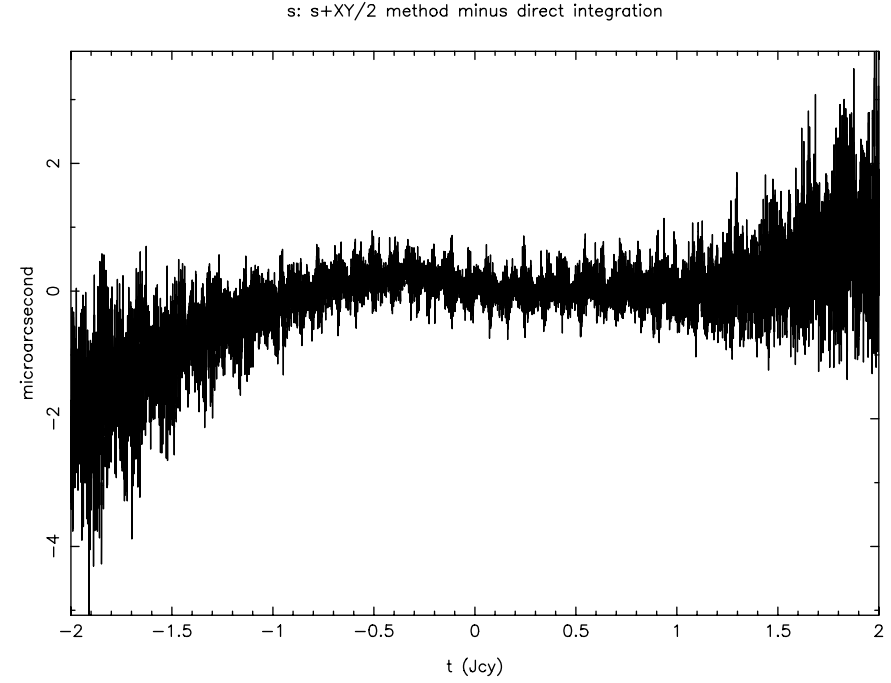

Fig. 5. Comparison between the CIO locator $s$ determined from the semi-analytical $s+X Y / 2$ series and from a direct numerical integration. See Sect. 5.2.

CIP change vector by writing $\Delta Z \simeq-(X \Delta X+Y \Delta Y) / Z$, and then rotate it using Eq. (25) with $\beta=s$. After eliminating insignificant terms, we find that:

$\Delta x \simeq \Delta X+\Delta X X^{2} / 2$,

$\Delta y \simeq \Delta Y+\Delta X(X Y / 2+s)$.

(An alternative is simply to rotate the new $X, Y, Z$ using the GCRS to CIRS matrix from the previous step of the numerical integration.)

To start the integration, we compute $\mathbf{M}(0)$, the GCRS to CIRS matrix for the CIP and CIO at epoch, using any of the methods described in Sect. 2. Then, for successive dates $t=\Delta t, 2 \Delta t \ldots$, we determine the GCRS coordinates $X, Y$ of the CIP, using any of the methods described in Sect. 3, and obtain $\Delta X, \Delta Y$ by differencing from the previous step. We use Eq. (82) to transform the changes to the CIRS and then Eq. (81) to apply the increment of rotation to $\mathbf{M}(t)$ to obtain $\mathbf{M}(t+\Delta t)$.

For any particular $\mathbf{M}(t)$ and corresponding $X, Y$, we can solve for $s$ as follows. Using Eq. (4) to evaluate $\mathbf{M}_{s=0}$, we can then use Eq. (3) to obtain:

$\mathbf{M}(t) \cdot \mathbf{M}_{s=0}^{T}=\left(\begin{array}{ccc}\cos s & -\sin s & 0 \\ \sin s & \cos s & 0 \\ 0 & 0 & 1\end{array}\right)$.

We then solve for $s$ by using either elements $(1,1)$ and $(1,2)$ or $(2,1)$ and $(2,2)$ and taking the arctangent.

This procedure was used to compute $s$ over a \pm 200 year test interval, using a time step of 0.1 days, for comparison with the predictions from the direct numerical integration of $s$ (Sect. 5.2). With the approximations shown, the results agreed to a few picoarcseconds throughout the test interval.

\subsection{End-to-end tests}

This paper has set out a number of ways of (i) locating the CIP and $\mathrm{CIO}$; (ii) forming the complete bias-precession-nutation 
rotation matrices; and (iii) calculating the associated Earth rotation measures. Trial software implementations of these algorithms were developed, both to validate the formulations and to assess their relative merits in various practical software roles.

Our approach was to implement the full GCRS to TIRS transformation by a variety of routes and, for test dates spanning four centuries centered on J2000, to see how well they agreed and to compare their computional efficiency. One of the methods, selected for its conciseness and rigor, was adopted as the reference. Numerical agreement was judged on the basis of the total rotational difference relative to the reference method: for two GCRS to TIRS matrices $\mathbf{R}_{\text {ref }}$ and $\mathbf{R}_{\text {test }}$, the rotational difference was defined as the rotation angle of the matrix $\mathbf{R}_{\text {ref }} \cdot \mathbf{R}_{\text {test }}^{T}$.

The specific objectives of the tests were, for each method:

1. Verify that the differences with respect to the reference method were satisfactorily small - no more than a few microseconds at the ends of the time span.

2. Assess the suitability for concise computer implementation using a procedural language such as Fortran.

3. Compare the computation time with that consumed by the reference method.

\subsubsection{Methods tested}

Seven different methods were tried, plus the adopted reference method:

Reference method: for the given date, compute the Fukushima-Williams precession angles (Sect. 2.2.3) and the MHB2000 nutation angles (luni-solar plus planetary), and form the NPB matrix (Eq. (15)). Compute the EO (Sect. 4.2) and the ERA, and set GST = ERA-EO. Use the third line of Eq. (62) to obtain the GCRS to TIRS matrix.

Method 1: compute $X, Y$ and $s+X Y / 2$ from series and form the NPB matrix (Sect. 2.1), then apply the ERA, i.e. the P03 equivalent of the technique described in IERS Conventions (2003).

Method 2: as for the reference method, compute the Fukushima-Williams precession angles and the MHB2000 nutation angles, then obtain $X, Y$ by evaluation of the corresponding two elements of the NPB matrix (Eq. (38)). Evaluate the $s+X Y / 2$ series (Sect. 4.1.2), obtain $s$ and evaluate the CIO based NPB matrix (Eq. (3)), then apply the ERA.

Method 3: as for the reference method, obtain the classical NPB matrix and EO, then use Eq. (75) to obtain $s$. Form the CIO based matrix (Eq. (3)) then compute and apply the ERA (first line of Eq. (62)).

Method 4: obtain the classical NPB matrix and EO as for the reference method. Use the EO to rotate the matrix onto the CIO and then apply ERA (Eq. (62), middle line).

Method 5: as for Method 1, obtain $X, Y$ from series, but instead of $s+X Y / 2$ use the $s+X Y / 2+D$ series (Eq. (61)) to obtain $s$, then construct the CIO based matrix using Eq. (3) and apply the ERA.

Method 6: evaluate the $x, y, z$ components of the equinox based $r$-vector (Sect. 2.3) from series and transform it into the

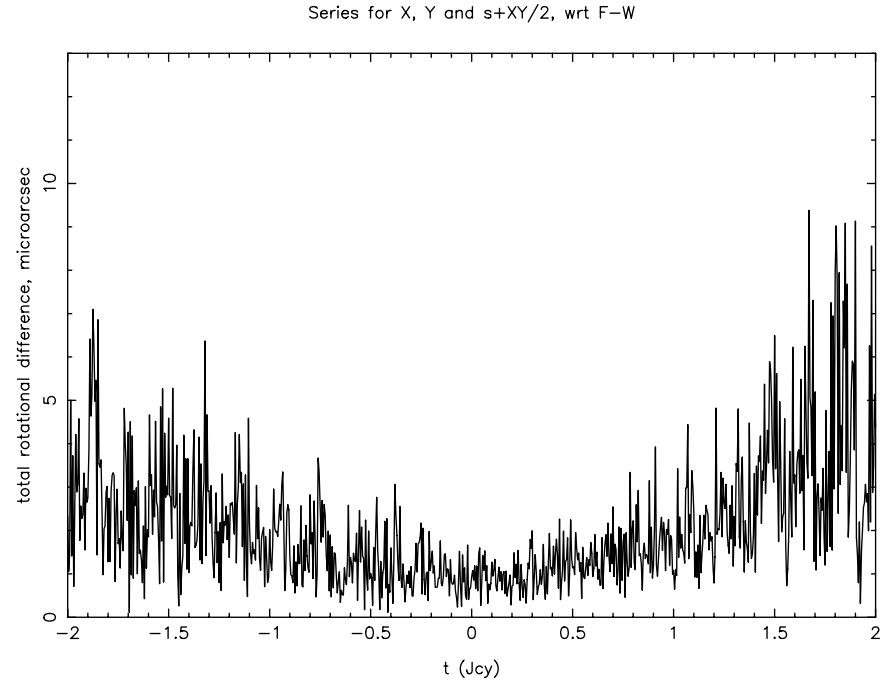

Fig. 6. The total rotational differences between the GCRS to TIRS matrices generated with the Fukushima-Williams/GST method and the $X, Y, s /$ ERA method (Method 1) from 1800 to 2200.

classical NPB matrix (Eqs. (16)-(18)). Compute the EO from series (Sect. EO), obtain GST from ERA-EO and form the GCRS to TIRS matrix (Eq. (62), bottom line).

Method 7: evaluate the $x, y, z$ components of the $\mathrm{CIO}$ based $r$-vector from series and transform it into the CIO based NPB matrix, then apply the ERA.

\subsubsection{Numerical agreement}

The rotational differences between the reference method (F-W, classical) and Method 1 ( $X, Y, s$ from series) are plotted in Fig. 6. At the ends of the time span the agreement is averaging about $5 \mu$ as. The results are dominated by the CIP position predicted by the respective algorithms.

For Method 2, $X$ and $Y$ are from the F-W matrix itself and the disagreement drops to the $1 \mu$ as level (Fig. 7). This time the differences are almost entirely due to the difference between the $s+X Y / 2$ and EO formulations.

Method 3 and Method 4 give essentially perfect results, limited mainly by rounding errors. In both cases the average errors are at the 20 pas level and are uniform across the time span.

Method 5 in effect tests the $s+X Y / 2+D$ series, delivering results of similar accuracy to Method 1 (Fig. 6), the latter based on the $s+X Y / 2$ series. The great similarity of the residuals with respect to the reference method shows that these two options for obtaining $s$ work equally well in the given context.

Methods 6 and 7 test the $r$-vector approach, with reference to the equinox and the $\mathrm{CIO}$ respectively. The results are quite similar, at $5 \mu$ as level at the ends of the time span, with the CIO variant (Fig. 8) marginally the better of the two.

\subsubsection{Software efficiency}

All the methods tested are based ultimately on the MHB2000 nutation model, which contains terms in various powers of $t$ 
$X, Y$ via $F / W$ matrix elements, then $s+X Y / 2$ series, wrt $F-W$

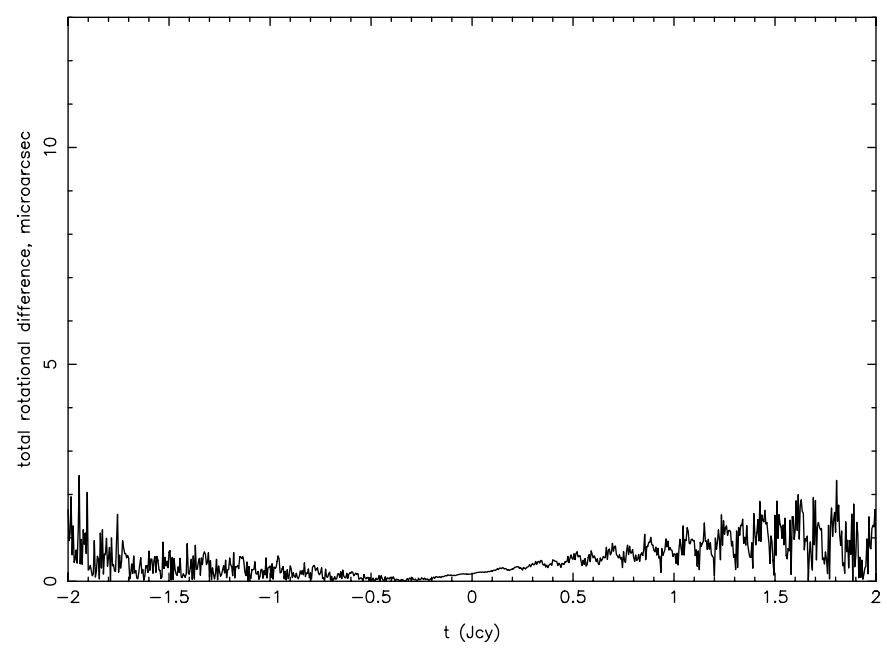

Fig. 7. The Method 2 residuals, which in effect compare the $s+X Y / 2$ and $\mathrm{EO}$ formulations.

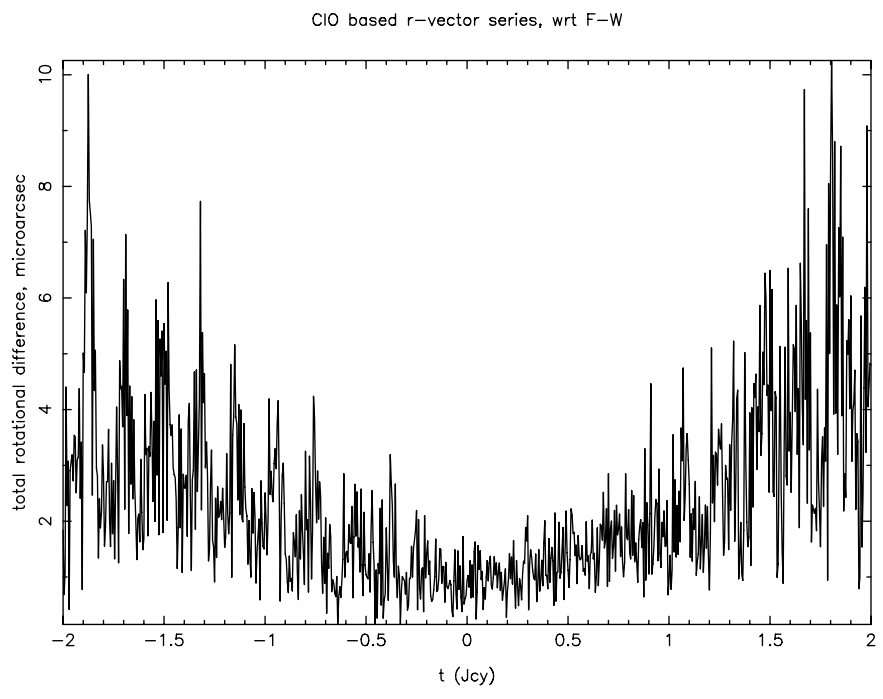

Fig. 8. Residuals from the $r$-vector series, CIO-based.

at 1365 different frequencies. This means that the computer code is in all cases dominated by nutation terms. Methods 2-5, plus the reference method, all use the MHB2000 nutation model itself, as implemented in the IAU SOFA routine i au_NUTOOA (Standards Of Fundamental Astronomy, Wallace 2002). The remainder, Methods 1, 6 and 7, implement the MHB2000 nutation in their own way. The computer code to be compared therefore comprises four Fortran subroutines, listed in Table 3. The execution times for the seven methods are shown in Table 4.

The quoted reductions in size are, if anything, conservative, because the three new algorithms provide frame bias and precession as well as nutation, though the latter dominates as already mentioned. The MHB2000 model is itself organized in an efficient way, to a sufficient extent to make the comparisons fair.

Note that Method 6, the equinox based $r$-vector method, is of theoretical interest only. Because the GST is required, and hence the equation of the equinoxes, the nutation com-
Table 3. The Fortran code used in the different methods tested. The line counts exclude blanks and comments. When writing these programs, an optimum balance was sought between efficiency and clarity.

\begin{tabular}{llc}
\hline \hline Algorithm & Lines & Methods \\
\hline iau_NUT00A & 3116 & ref. 2-5 \\
$X, Y$ series & 2363 (24\% smaller) & 1 \\
$r$-vector series, equinox & $2530(19 \%$ smaller $)$ & 6 \\
$r$-vector series, CIO & $2432(22 \%$ smaller $)$ & 7 \\
\hline
\end{tabular}

Table 4. The execution speed of the different methods. The figures are for generating the full GCRS to TIRS matrix, i.e. they include the computation of either GST or ERA.

\begin{tabular}{cc}
\hline \hline Method & Speed \\
\hline reference & $100 \%$ \\
1 & $16 \%$ faster \\
2 & $\simeq$ \\
3 & $\simeq$ \\
4 & $\simeq$ \\
5 & $\simeq$ \\
6 & half as fast \\
7 & $23 \%$ faster \\
\hline
\end{tabular}

ponent $\Delta \psi$ is needed, which would mean evaluating the MHB2000 nutation model as well as the $r$-vector series. This extra overhead explains why Method 6 took twice as long as the others and rules out its practical use.

\section{Summary and conclusions}

We have described a number of approaches to forming the precession-nutation transformation and various ways of locating the pole and longitude origin. We have reported on both the real accuracy and the computational precision that can be achieved, and have assessed the suitability of the different methods for high-accuracy applications in the future. These include applications requiring high-accuracy knowledge of the Earth's orientation in space and scientific studies for improving the dynamical model of Earth's rotation by comparing predictions to VLBI observations.

First, we have confirmed that the use of the CIO paradigm, that keeps the concept of the pole position and Earth rotation separate, leads to a cleaner implementation that has particular advantages for high accuracy Earth rotation studies.

Second, we have shown that using the EO clarifies the differences between the classical and CIO-based transformations, for example by showing how ERA and the IAU 2000 GST(UT1) are related.

Third, as users of the classical method should benefit from the full accuracy of the latest models, we have tried to ensure with the GCRS to TIRS transformation the equinox based method has the same accuracy as that achievable with the CIO based method. 
Fourth, we have confirmed that the bias-precessionnutation F-W angles lead to the most concise expressions for the CIP $X, Y$ coordinates, given the current form of the precession-nutation models, and provide a simple link between the classical and new methods.

Fifth, we have investigated how to express the complete NPB transformation as the three numbers making up the rotation vector (Sects. 2.4.3 and 2.3). Although this scheme is not efficient in all cases, series for the CIO based $r$-vector $x$, $y$ and $z$ components produce a result that is quite competitive with any of the other methods, and this may influence the development of future models and applications.

We have in particular showed the following:

(i) Once the parameters of the precession-nutation model are chosen, the semi-analytical series for calculating the CIP and CIO directions obtained by several different methods all agree at a submicroarcsecond level.

(ii) The difference between the predictions of the CIP $X, Y$ from semi-analytical series and by evaluating the corresponding elements of the classical NPB matrix are of the order of $5 \mu$ as after 2 centuries, which meets the requirements of high-accuracy applications. Note, however, that due to the uncertainties in the precession-nutation model parameters, the real accuracy that can be achieved in the predicted CIP location is only of the order of 2 mas.

(iii) The differences between the predictions of the series for the $\mathrm{CIO}$ direction and numerical integration are of the order of $5 \mu$ as after 2 centuries, which meets the requirements of high-accuracy applications.

(iv) Given the known uncertainties in the current precessionnutation model, the GCRS direction of the CIO can be predicted, by a variety of methods, to an accuracy of a few microarcseconds. The equation of the origins is several orders of magnitude more dependent on the model; however, as the resulting uncertainties are in the equinox position rather than the CIO position, the $\mathrm{EO}$ can be used to locate the latter to a comparable accuracy to that from the other methods provided that formulations compatible at microarcsecond level be used for the EO and equinox based NPB.

(v) The differences between the various ways of implementing the full GCRS to TIRS transformation are of the order of $5 \mu$ as after 2 centuries.

Acknowledgements. We are grateful to the referee of this paper, Jan Vondrák, for his helpful suggestions for improving the manuscript.

\section{Appendix A: the electronic tables}

Tables 5-11 for the complete series developments for the quantities $s$ (Eq. (53)), $s+X Y / 2$ (Eq. (58)), $s+X Y / 2+D$ (Eq. (60)), $\mathrm{EO}+\Delta \psi \cos \epsilon_{A}$ (where $\mathrm{EO}$ is given by Eq. (69)), $x_{\mathrm{CIO}}, y_{\mathrm{CIO}}, z_{\mathrm{CIO}}$ (Eq. (70)), respectively, retaining all terms larger than $0.1 \mu$ as, are available in electronic form at the CDS.
These tables represent the Fourier and Poisson terms of the series, as output by the GREGOIRE software. The general formula is:

$$
\begin{aligned}
S= & \sum_{\left(i_{1}, i_{2}, \ldots, i_{n}\right)} \sum_{k=0}^{p} C_{i_{1}, i_{2}, \ldots, i_{n}}^{(k)} t^{k} \cos \left(i_{1} y_{1}+i_{2} y_{2}+\ldots+i_{n} y_{n}\right) \\
& +S_{i_{1}, i_{2}, \ldots, i_{n}}^{(k)} t^{k} \sin \left(i_{1} y_{1}+i_{2} y_{2}+\ldots+i_{n} y_{n}\right)
\end{aligned}
$$

where $t$ is the time as given by Eq. (9), the upper limit $p$ of the powers of $t$ in the Poisson terms is equal to 5 , and the quantities $C_{i_{1}, i_{2}, \ldots, i_{n}}^{(k)}$ and $S_{i_{1}, i_{2}, \ldots, i_{n}}^{(k)}$ are numerical coefficients which are limited by a precision $\epsilon$, equal to $0.1 \mu$ as, by omitting terms where

$\left|C_{i_{1}, i_{2}, \ldots, i_{n}}^{(k)}\right|+\left|S_{i_{1}, i_{2}, \ldots, i_{n}}^{(k)}\right|<\epsilon$.

The arguments of the series have formal components described by the set of integers $\left(i_{1}, i_{2}, \ldots, i_{n}\right)$, with $n=14$, and the fundamental lunisolar and planetary arguments of the nutation theory.

\section{References}

Aoki, S., \& Kinoshita, H. 1983, Celest. Mech., 29, 335

Capitaine, N., \& Gontier, A.-M. 1993, A\&A, 275, 645

Capitaine, N., Guinot, B., \& Souchay, J. 1986, Celest. Mech., 39, 283

Capitaine, N., Chapront, J., Lambert, S., \& Wallace, P. T. 2003a, A\&A, 400, 1145 (C03a)

Capitaine, N., Wallace, P. T., \& McCarthy, D. D. 2003b, A\&A, 406, 1135 (C03b)

Capitaine, N., Wallace, P. T., \& Chapront, J. 2003c, A\&A, 412, 567 (C03c)

Capitaine, N., Wallace, P. T., \& Chapront, J. 2005, A\&A, 432, 355 (C05)

Capitaine, N., et al. 2006, in Reports on Astronomy 2002-2005, IAU Transaction XXVIA, 2006, ed. O. Engvold http://syrte.obspm.fr/iauWGnfa/

Chapront, J. 2003, Notice, Paris Observatory, January 2003

Chapront, J., Chapront-Touzé, M., \& Francou, G. 2002, A\&A, 387, 700

Fukushima, T. 2001, AJ, 122, 482

Fukushima, T. 2003, AJ, 126, 494

Fukushima, T. 2004, in Proc. of the Journées Systèmes de référence spatio-temporels 2003, ed. A. Finkelstein, \& N. Capitaine, St. Petersburg, 114

Goldstein, H. 1980, Classical Mechanics 2nd edn. (Addison Wesley)

Guinot, B. 1979, in Time and the Earth's Rotation, ed. D. D. McCarthy, \& J. D. Pilkington (D. Reidel Publishing Company), 7

Hilton, J., et al. 2006, in Reports on Astronomy 2002-2005, IAU Transaction XXVIA, ed. O. Engvold

IERS Conventions 2003, IERS Technical Note 32, ed. D. D. McCarthy, \& G. Petit, Frankfurt am Main: Verlag des Bundesamts für Kartographie und Geodäsie, 2004

Kaplan, G. 2003, Another Look at Non-rotating Origins, to appear in the Proceedings of Joint Discussion 16, IAU 2003 GA

Mathews, P. M., Herring, T. A., \& Buffett, B. A. 2002, J. Geophys. Res., 107, B4

Vondrák, J., Ron, C., \& Weber, R. 2003, A\&A, 397, 771

Wallace, P. T. 2002, Highlights of Astronomy, ed. H. Rickman (Astronomical Society of the Pacific), 12, 128

Wertz, J. M. 1986, Spacecraft Attitude Determination and Control, ed. J. R. Wertz, Astrophysics and Space Science Library, 73 (D. Reidel Publishing Company), 1986

Williams, J. G. 1994, AJ, 108(2), 711 